\title{
Equivariant $K$-theory, generalized symmetric products, and twisted Heisenberg algebra
}

\author{
Weiqiang Wang*
}

\begin{abstract}
For a space $X$ acted by a finite group $\Gamma$, the product space $X^{n}$ affords a natural action of the wreath product $\Gamma_{n}=\Gamma^{n} \rtimes S_{n}$. The direct sum of equivariant $K$-groups $\bigoplus_{n \geq 0} K_{\Gamma_{n}}\left(X^{n}\right) \otimes \mathbb{C}$ were shown earlier by the author to carry several interesting algebraic structures. In this paper we study the $K$ groups $K_{\widetilde{H} \Gamma_{n}}\left(X^{n}\right)$ of $\Gamma_{n}$-equivariant Clifford supermodules on $X^{n}$. We show that $\mathcal{F}_{\Gamma}^{-}(X)=\bigoplus_{n \geq 0} K_{\widetilde{H} \Gamma_{n}}\left(X^{n}\right) \otimes \mathbb{C}$ is a Hopf algebra and it is isomorphic to the Fock space of a twisted Heisenberg algebra. Twisted vertex operators make a natural appearance. The algebraic structures on $\mathcal{F}_{\Gamma}^{-}(X)$, when $\Gamma$ is trivial and $X$ is a point, specialize to those on a ring of symmetric functions with the Schur $Q$-functions as a linear basis. As a by-product, we present a novel construction of $K$-theory operations using the spin representations of the hyperoctahedral groups.
\end{abstract}

\section{Contents}

1 Introduction 2

2 The group $\widetilde{H} \Gamma_{n}$ and its spin supermodules 5

2.1 Definition of the supergroup $\widetilde{H} \Gamma_{n} \ldots \ldots \ldots \ldots$

2.2 Conjugacy classes of $\widetilde{H} \Gamma_{n} \ldots \ldots \ldots \ldots$. . . . . . . . . . . . . . . . . . 7

2.3 Spin supermodules over $\widetilde{H} \Gamma_{n} \ldots \ldots \ldots$. . . . . . . . . . . . . . . 9

3 A decomposition theorem in equivariant $K$-theory 11

3.1 The standard version . . . . . . . . . . . . . . . . . . . 11

3.2 A super $/$ twisted variant . . . . . . . . . . . . . . . 12

4 The group $\widetilde{H}_{n}$ and $K$-theory operations 14

4.1 The group $\widetilde{H}_{n}$ and a ring of symmetric functions . . . . . . . . . . . 14

4.2 K-theory operations . . . . . . . . . . . . . . . . 16

*Partially supported by an NSF grant and an FR\&PD grant at NCSU. 


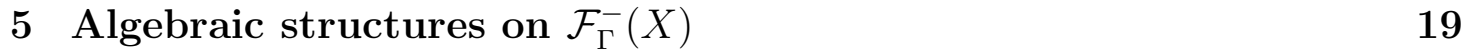

5.1 Generalized symmetric products . . . . . . . . . . . . . . . . 19

5.2 Hopf algebra $\mathcal{F}_{\Gamma}^{-}(X) \ldots \ldots \ldots \ldots \ldots$

5.3 Description of the algebra $\mathcal{F}_{\Gamma}^{-}(X) \ldots \ldots \ldots \ldots \ldots \ldots$

5.4 Twisted vertex operators and $\mathcal{F}_{\Gamma}^{-}(X) \ldots \ldots \ldots \ldots$

5.5 Twisted Heisenberg algebra and $\mathcal{F}_{\Gamma}^{-}(X) \ldots \ldots \ldots \ldots$

6 Appendix: Another formulation using $\widetilde{S}_{n}$ and $\widetilde{\Gamma}_{n} \quad 28$

\section{Introduction}

Motivated in part by Vafa-Witten [VW1] and generalizing the work of Segal [Seg2] (also cf. Grojnowski [Gr]), we studied in [W1] a direct sum, denoted by $\mathcal{F}_{\Gamma}(X)$, of the equivariant $K$-groups $\oplus_{n \geq 0} K_{\Gamma_{n}}\left(X^{n}\right) \otimes \mathbb{C}$ associated to a topological $\Gamma$-space $X$. Here $\Gamma$ is a finite group and the wreath product $\Gamma_{n}$ (i.e. the semi-direct product $\Gamma^{n} \rtimes S_{n}$ ) acts naturally on the $n$th Cartisian product $X^{n}$. We proved that the space $\mathcal{F}_{\Gamma}(X)$ carries several remarkable algebraic structures such as Hopf algebra and Fock representation of a Heisenberg (super)algebra etc, and that vertex operators makes a natural appearance as a part of $\lambda$-ring structure. We in addition pointed out in [W1] a new approach to the realization of the Frenkel-Kac-Segal homogeneous vertex representations of affine Lie algebras by using representation rings of the wreath product $\Gamma_{n}$ associated to a finite subgroup $\Gamma$ of $S L_{2}(\mathbb{C})$. This has been subsequently completed in [FJW1] jointly with I. Frenkel and Jing, and further extended in [FJW2] to realize vertex representations of twisted affine and toroidal Lie algebras by using the spin representation rings of a double cover $\widetilde{\Gamma}_{n}$ of the wreath product $\Gamma_{n}$.

In this paper we will introduce a variant of equivariant $K$-theory. Given a topological space $X$ acted upon by a finite supergroup $G$ in an appropriate sense, we introduce a category of complex $G$-equivariant spin vector superbundles over $X$, and consider the corresponding Grothendieck group $K_{G}^{-}(X)$. The superscript here and below is used in this paper to stand for spin, i.e. a certain central element $z$ in the supergroup $G$ acts as -1 . This $K$-group can be thought as an invariant of orbifolds with (certain distinguished) discrete torsion as introduced by Vafa [Va]. Discrete torsion has been a topic of interest from various viewpoints since then, cf. [VW2, Di, AR, Ru, Sh] and the references therein. We present here a variant of the decomposition theorem of Adem and Ruan [AR] for what they call twisted equivariant $K$-theory, which generalizes the decomposition theorem [BC, $\mathrm{Ku}$ ] (also cf. [AS, HKR]) in equivariant $K$-theory.

Our formulation of such $K$-groups is motivated by providing a general framework for the main subject of study in this paper, namely a spin/twisted version of the space $\mathcal{F}_{\Gamma}(X)$ studied in [W1]. When the topological space under consideration is a point, our $K$-theory specializes to the theory of supermodules of finite super- 
groups, cf. Józefiak [Jo1]. (In this paper super always means $\mathbb{Z}_{2}$-graded). Such a theory of supermodules has provided, in our opinion, a most natural framework (cf. [Jo]) for the exposition of the spin representations of a double cover $\widetilde{S}_{n}$ of the symmetric group initiated by Schur [Sc]. The spin representation theory of a double cover $\widetilde{H}_{n}$ of the hyperoctahedral group, being almost parallel to and to some extent simpler than the spin representation theory for $\widetilde{S}_{n}$, can also be treated successfully in terms of supermodules (cf. [Jo2]).

Given a space $X$ acted by a (non-graded) finite group $\Gamma$, we obtain our main example of such $K$-group $K_{\widetilde{H} \Gamma_{n}}^{-}\left(X^{n}\right)$ by considering the action on the $n$-th Cartesian product $X^{n}$ by the wreath product $\Gamma_{n}$ which is further extended trivially to the action of a larger finite supergroup $\widetilde{H} \Gamma_{n}$. Here $\widetilde{H} \Gamma_{n}$ is a double cover of the wreath product $\left(\Gamma \times \mathbb{Z}_{2}\right)^{n} \rtimes S_{n}$. (We recommend that the reader sets $\Gamma$ to be the one-element group in their first reading so that the whole picture becomes simpler and more transparent. In this case $\widetilde{H} \Gamma_{n}$ reduces to a double cover $\widetilde{H}_{n}$ of the Hyperoctahedral group.) The category of $\widetilde{H} \Gamma_{n}$-equivariant spin vector superbundles over $X^{n}$ admits an equivalent reformulation which has a perhaps better geometric meaning. Namely this is the category of $\Gamma_{n}$-equivariant vector bundles $E$ over $X^{n}$ such that $E$ carries a supermodule structure with respect to the complex Clifford algebra of rank $n$ which is compatible with the action of $\Gamma_{n}$.

A fundamental example of $\widetilde{H} \Gamma_{n}$-vector superbundles over $X^{n}$, which plays an important role in this paper, is given as follows for $X$ compact. Given a $\Gamma$-vector bundle $V$ over $X$, we consider the vector superbundle $V \mid V=V \oplus V$ over $X$ with the natural $\mathbb{Z}_{2}$-grading. We can endow the $n$th outer tensor $(V \mid V)^{\otimes n}$ a natural $\widetilde{H} \Gamma_{n}$-equivariant vector superbundle structure over $X^{n}$.

We will show that the direct sum

$$
\mathcal{F}_{\Gamma}^{-}(X):=\bigoplus_{n=0}^{\infty} K_{\widetilde{H} \Gamma_{n}}^{-}\left(X^{n}\right) \bigotimes \mathbb{C}
$$

carries naturally a Hopf algebra structure and it is isomorphic to the Fock space of a twisted Heisenberg superalgebra associated to $K_{\widetilde{H} \Gamma_{1}}^{-}(X) \cong K_{\Gamma}(X)$. All the algebraic structures are constructed in terms of natural $K$-theory maps. In particular the dimension of $K_{\widetilde{H} \Gamma_{n}}\left(X^{n}\right)$ is determined explicitly for all $n$. We remark that such a twisted Heisenberg algebra has played an important role in the theory of affine Kac-Moody algebras, cf. [FLM]. Roughly speaking, the structure of the space $\mathcal{F}_{\Gamma}(X)$ studied in [Seg2, W1] is modeled on the ring $\Lambda_{\mathbb{C}}$ of symmetric functions with a basis given by Schur functions (or equivalently on the direct sum of representation rings of symmetric groups $S_{n}$ for all $n$ ). The structure of the space $\mathcal{F}_{\Gamma}^{-}(X)$ under consideration of this paper is shown to be modeled instead on the ring $\Omega_{\mathbb{C}}$ of symmetric functions with a linear basis given by the so-called Schur $Q$-functions (or equivalently on the direct sum of the spin representation ring of $\widetilde{H}_{n}$ for all $n$ ). It is well known that the graded dimension of the ring $\Omega_{\mathbb{C}}$ is given 
by the generating function

$$
\frac{1}{\prod_{i=0}^{\infty}\left(1-q^{2 i-1}\right)}=q^{-\frac{1}{24}} \frac{\eta\left(q^{2}\right)}{\eta(q)}
$$

where $\eta(q)$ is the Dedekind $\eta$ function.

Just as the $\lambda$ ring is modeled on the ring $\Lambda_{\mathbb{C}}$ of symmetric functions (cf. e.g. $[\mathrm{Kn}])$, one can introduce a $Q-\lambda$ ring $^{1}$ structure modeled on the ring $\Omega_{\mathbb{C}}$ with Adams operations of odd degrees only. We show that as a part of the $Q-\lambda$ ring structure on $\mathcal{F}_{\Gamma}^{-}(X)$ twisted vertex operators naturally appear in $\mathcal{F}_{\Gamma}^{-}(X)$ via the $n$-th outer tensor $(V \mid V)^{\otimes n}$ associated to $V \in K_{\Gamma}(X)$ in terms of the Adams operations.

It is of independent interest to see that when we restrict $K_{\widetilde{H}_{n}}^{-}\left(X^{n}\right)$ from $X^{n}$ to its diagonal we are able to obtain various $K$-theory operations on $K(X)$, including supersymmetric power operations and Adams operations of odd degrees, by means of the spin supermodules of $\widetilde{H}_{n}$. This is a super analog of Atiyah's construction [At] of $K$-theory operations on $K(X)$ by means of the representations of the symmetric groups.

Motivated by Göttsche's formula, Vafa and Witten [VW1] conjectured that the direct sum $\mathcal{H}(S)$ of homology groups for Hilbert schemes $S^{[n]}$ of $n$ points on a (quasi-)projective surface $S$ should carry the structure of a Fock space of a Heisenberg algebra, which was realized subsequently in a geometric way by Nakajima and Grojnowski (cf. [Na, Gr]). Parallel algebraic structures such as Hopf algebra, vertex operators, and Heisenberg algebra as part of vertex algebra structures [Bo, FLM], have naturally showed up in $\mathcal{H}(S)$ as well as in $\mathcal{F}_{\Gamma}(X)$. When $S$ is a suitable resolution of singularities of an orbifold $X / \Gamma$, there appears close connections between $\mathcal{H}(S)$ and $\mathcal{F}_{\Gamma}(X)$, cf. [W2, W3] and the references therein. It will be very important to see if one can find a 'Hilbert scheme' version of the orbifold picture drawn in this paper. It is also interesting to see if our current work can find some applications in string theory, cf. [VW1, VW2, Di, Sh]. In fact the special case of our construction for $\Gamma$ trivial is closely related to an earlier paper of Dijkgraaf [Di]. See the Appendix.

When $X$ is a point, the $K$-group $\underline{K}_{\widetilde{H} \Gamma_{n}}^{-}\left(X^{n}\right)$ becomes the Grothendieck group of spin supermodules of $\widetilde{H} \Gamma_{n}$. In a companion paper [JW] joint with Jing, the conjugacy classes and Grothendieck groups of $\widetilde{H} \Gamma_{n}$ have been studied in detail. In particular, when $\Gamma$ is a subgroup of $S L_{2}(\mathbb{C})$, they are used to realize vertex representations of twisted affine algebras (cf. [FLM]) and toroidal Lie algebras. This provides a new group theoretic construction, a somewhat improved version in our opinion than the one in [FJW2] using the groups $\widetilde{\Gamma}_{n}$, of twisted vertex representations. In the Appendix, we sketch another formulation of our main results in this paper using the groups $\widetilde{S}_{n}$ and $\widetilde{\Gamma_{n}}$ instead of $\widetilde{H}_{n}$ and $\widetilde{H} \Gamma_{n}$.

\footnotetext{
${ }^{1}$ Here $Q$ stands for Queer or Schur $Q$-functions. We believe that there exists a rich $Q$ mathematical world which is relevant to various twisted, spin, super structures, etc.
} 
The plan of this paper is as follows. In Sect. 2 we present the spin representation rings of the finite supergroup $\widetilde{H} \Gamma_{n}$. In Sect. 3 we introduce the category and $K$ groups of spin vector superbundles which are equivariant with respect to finite supergroups and present a decomposition theorem for such equivariant $K$-groups. In Sect. 4 we study $K$-theory operations based on the spin representations of $\widetilde{H}_{n}$. The results in this section are not to be further used in this paper. In Sect. 5, which is the heart of the paper, we present the structures of a Hopf algebra and of a $Q-\lambda$ ring on $\mathcal{F}_{\Gamma}^{-}(X)$, and relate the latter to the twisted vertex operators. We further construct a Heisenberg algebra which acts on $\mathcal{F}_{\Gamma}^{-}(X)$ irreducibly by means of natural $K$-theory maps. In the Appendix, we outline a somewhat different construction in terms of the group $\widetilde{\Gamma_{n}}$.

\section{The group $\widetilde{H} \Gamma_{n}$ and its spin supermodules}

In this section we recall briefly some essential points in the theory of supermodules of a finite supergroup (cf. [Jo1]). We define the finite supergroup $\widetilde{H} \Gamma_{n}$ associated to any finite group $\Gamma$, and study its conjugacy classes and spin supermodules. More detail of these can be found in $[\mathrm{JW}]$.

\subsection{Definition of the supergroup $\widetilde{H} \Gamma_{n}$}

Let $\widetilde{G}$ be a finite group and let $d: \widetilde{G} \rightarrow \mathbb{Z}_{2}$ be a group epimorphism. We denote by $\widetilde{G}_{0}$ the kernel of $d$ which is a subgroup of $\widetilde{G}$ of index 2 . We regard $d(\cdot)$ as a parity function on $\widetilde{G}$ by letting the degree of elements in $\widetilde{G}_{0}$ be 0 and the degree of elements in the complementary $\widetilde{G}_{1}=\widetilde{G} \backslash \widetilde{G}_{0}$ be 1 . Elements in $\widetilde{G}_{0}$ (resp. $\widetilde{G}_{1}$ ) will be called even (resp. odd). We will often refer to the pair $(\widetilde{G}, d)$, or simply $\widetilde{G}$ when there is no ambiguity, as a finite supergroup. The class of finite supergroups under consideration in this paper has an additional property: it contains a distinguished even central element $z$ of order 2 . We denote by $\theta$ the quotient group homomorphism $\widetilde{G} \rightarrow G \equiv \widetilde{G} /\langle 1, z\rangle$.

In this paper the modules over a finite supergroup or a superalgebra (such as the group superalgebra of a finite supergroup) will always be $\mathbb{Z}_{2}$-graded (i.e. supermodules) unless otherwise specified. A general theory of supermodules over finite supergroups was developed by Józefiak [Jo1]. This was motivated to provide a modern account [Jo] of Schur's seminal work on spin representations of symmetric groups $[\mathrm{Sc}]$.

Given two supermodules $M=M_{0}+M_{1}$ and $N=N_{0}+N_{1}$ over a superalgebra $A=A_{0}+A_{1}$, the linear map $f: M \rightarrow N$ between two $A$-supermodules is a homomorphism of degree $i$ if $f\left(M_{j}\right) \subset M_{i+j}$ and for any homogeneous element $a \in A$ and any homogeneous vector $m \in M$ we have

$$
f(a m)=(-1)^{d(f) d(a)} a f(m) .
$$


The degree 0 (resp. 1) part of a superspace is referred to as the even (resp. odd) part. We denote

$$
\operatorname{Hom}_{A}(M, N)=\operatorname{Hom}_{A}(M, N)_{0} \oplus \operatorname{Hom}_{A}(M, N)_{1},
$$

where $\operatorname{Hom}_{A}(M, N)_{i}$ consists of $A$-homomorphisms of degree $i$ from $M$ to $N$. The notions of submodules, tensor product, and irreducibility etc for supermodules are defined similarly.

Given a finite supergroup $\widetilde{G}$, a $\widetilde{G}$-supermodule $V$ is called spin if the central element $z$ acts as -1 . Alternatively, one can associate a 2-cocycle $\alpha: G \times G \rightarrow \mathbb{Z}_{2}=$ $\{ \pm 1\}$, such that $V$ becomes a projective supermodule of the group $G=\widetilde{G} /\langle 1, z\rangle$ associated with $\alpha$, namely, the actions of any two elements $g, h \in G$ on $V$, denoted by $\rho(g), \rho(h)$, satisfy the relation

$$
\rho(g) \rho(h)=\alpha(g, h) \rho(g h)
$$

Among all $\widetilde{G}$-supermodules, we will only consider the spin supermodules in this paper. It is clear that the restriction (resp. the induction) of a spin supermodule to a $\mathbb{Z}_{2}$-graded subgroup (resp. a larger supergroup) with the same distinguished even element $z$ remains to be a spin supermodule.

There are two types of complex simple superalgebras according to C.T.C. Wall: $M(r \mid s)$ and $Q(n)$. Here $M(r \mid s)$ is the superalgebra consisting of the linear transformations of the superspace $\mathbb{C}^{r \mid s}=\mathbb{C}^{r}+\mathbb{C}^{s}$. The superalgebra $Q(n)$ is the graded subalgebra of $M(n \mid n)$ consisting of matrices of the form

$$
\left[\begin{array}{ll}
A & B \\
B & A
\end{array}\right] \text {. }
$$

It is known [Jo1] that the group (super)algebra of a finite supergroup is semisimple, i.e. decomposes into a direct sum of simple superalgebras. According to the classification of simple superalgebras above, the irreducible supermodules of a finite supergroup are divided into two types, type $M$ and type $Q$. We note that the endomorphism algebra of an irreducible supermodule $V$ is isomorphic to $\mathbb{C}$ if $V$ is of type $M$ and isomorphic to the complex Clifford algebra $C_{1}$ in one variable if $V$ is of type $Q$.

Let $\Pi_{n}$ the group generated by $1, z, a_{1}, \ldots, a_{n}$ subject to the relations

$$
z^{2}=1, a_{i}^{2}=z \text { and } a_{i} a_{j}=z a_{j} a_{i} \text {, for } i \neq j .
$$

The symmetric group $S_{n}$ acts on $\Pi_{n}$ via permutations of the elements $a_{1}, \ldots, a_{n}$, i.e. $\sigma\left(a_{i}\right)=a_{\sigma(i)}$ for $\sigma \in S_{n}$. We thus form the semi-direct product $\widetilde{H}_{n}=\Pi_{n} \rtimes S_{n}$, which naturally endows a $\mathbb{Z}_{2}$-grading given by the parity $d\left(a_{i}\right)=1, d(z)=0$, and $d(\sigma)=0$ for $\sigma \in S_{n}$. We may therefore regard $\widetilde{H}_{n}$ as a finite supergroup with a distinguished even central element. Note that the group superalgebra $\mathbb{C}\left[\widetilde{H}_{n}\right] /\langle z=-1\rangle$ is exactly the complex Clifford algebra $C_{n}$ in $n$ variables. 
Let $\Gamma$ be a finite group with $r+1$ conjugacy classes. We denote by $\Gamma^{*}=\left\{\gamma_{i}\right\}_{i=0}^{r}$ the set of complex irreducible characters where $\gamma_{0}$ denotes the trivial character, and by $\Gamma_{*}$ the set of conjugacy classes. Let $R(\Gamma)=\oplus_{i=0}^{r} \mathbb{C} \gamma_{i}$ be the space of class functions on $\Gamma$, and set $R_{\mathbb{Z}}(\Gamma)=\oplus_{i=0}^{r} \mathbb{C} \gamma_{i}$. For $c \in \Gamma_{*}$ let $\zeta_{c}$ be the order of the centralizer of an element in the conjugacy class $c$, so the order of the class is then $|\Gamma| / \zeta_{c}$

Given a positive integer $n$, let $\Gamma^{n}=\Gamma \times \cdots \times \Gamma$ be the $n$-th direct product of $\Gamma$, and let $\Gamma^{0}$ be the trivial group. The symmetric group $S_{n}$ naturally acts on $\Gamma^{n} \times \Pi_{n}$ by simultaneous permutations of elements in $\Gamma^{n}$ and $\Pi_{n}$.

The finite supergroup $\widetilde{H} \Gamma_{n}$ is then defined to be the semi-direct product of the symmetric group $S_{n}$ and $\Gamma^{n} \times \Pi_{n}$, with the multiplication given by

$$
(g, \sigma) \cdot(h, \tau)=(g \sigma(h), \sigma \tau), \quad g, h \in \Gamma^{n} \times \Pi_{n}, \sigma, \tau \in S_{n}
$$

The order of $\widetilde{H} \Gamma_{n}$ is clearly $2^{n+1} n !|\Gamma|^{n}$. The $\mathbb{Z}_{2}$-grading on $\widetilde{H} \Gamma_{n}$ is induced from that on $\Pi_{n}$ and by letting the elements in $\Gamma^{n}$ be even (i.e. of degree 0 ). Denoting by $H \Gamma_{n}=\left(\Gamma \times \mathbb{Z}_{2}\right)^{n} \rtimes S_{n}$, we have the following exact sequence of groups:

$$
1 \rightarrow \mathbb{Z}_{2}=\{1, z\} \rightarrow \widetilde{H} \Gamma_{n} \stackrel{\theta_{n}}{\rightarrow} H \Gamma_{n} \rightarrow 1
$$

It is clear that when $\Gamma$ is trivial $\widetilde{H} \Gamma_{n}$ reduces to a double cover $\widetilde{H}_{n}$ of the hyperoctahedral group $H_{n}=\mathbb{Z}_{2}^{n} \rtimes S_{n}$.

The finite supergroup $\widetilde{H} \Gamma_{n}$ contains $\Pi_{n}, \widetilde{H}_{n}$ and the wreath product $\Gamma_{n}=$ $\Gamma^{n} \rtimes S_{n}$ as distinguished subgroups. Letting $\Gamma^{n}$ act trivially on $\Pi_{n}$ we extend the action of the symmetric group $S_{n}$ to $\Gamma_{n}$ on $\Pi_{n}$. In this way we may also view $\widetilde{H} \Gamma_{n}$ as a semi-direct product between $\Gamma_{n}$ and $\Pi_{n}$.

\subsection{Conjugacy classes of $\widetilde{H} \Gamma_{n}$}

Let $\widetilde{G}$ be a finite supergroup and put $G=\widetilde{G} /\langle 1, z\rangle$ and $\theta: \widetilde{G} \rightarrow G$ as before. For any conjugacy class $C$ of $G, \theta^{-1}(C)$ is either a conjugacy class of $\widetilde{G}$ or it splits into two conjugacy classes of $\widetilde{G}$, cf. [Jo1]. If $\theta^{-1}(C)$ splits, the conjugacy class $C$ will be referred to as split, and an element $g$ in $C$ is also called split, which is equivalent to say that the two preimages of $g$ under $\theta$ are not conjugate to each other. Otherwise $g$ is said to be non-split. In view of (1), we have the following easy equivalent formulation.

Lemma 2.1 An element $g$ in $G=\widetilde{G} /\langle 1, z\rangle$ is split if and only if

$$
\varepsilon_{g}(\cdot):=\alpha(g, \cdot) \alpha(\cdot, g)^{-1}
$$

defines a trivial character of the centralizer group $Z_{G}(g)$. 
For the study of spin supermodules of $\widetilde{H} \Gamma_{n}$, it is crucial to have a detailed description of split conjugacy classes of $\widetilde{G}$. Indeed the characters of spin supermodules vanish on non-split conjugacy classes as well as on odd split classes, cf. [Jo1]. Below we will concentrate on the group $\widetilde{H} \Gamma_{n}$.

Let $\lambda=\left(\lambda_{1}, \lambda_{2}, \cdots, \lambda_{l}\right)$ be a partition of integer $|\lambda|=\lambda_{1}+\cdots+\lambda_{l}$, where $\lambda_{1} \geq \ldots \geq \lambda_{l} \geq 1$. The integer $l$ is called the length of the partition $\lambda$ and is denoted by $l(\lambda)$. We will also make use of another notation for partitions:

$$
\lambda=\left(1^{m_{1}} 2^{m_{2}} \cdots\right),
$$

where $m_{i}$ is the number of parts in $\lambda$ equal to $i$. A partition $\lambda$ is strict if its parts are distinct integers, namely all the multiplicities $m_{i}$ are 1 or 0 . Given a partition $\lambda=\left(1^{m_{1}} 2^{m_{2}} \ldots\right)$ of $n$, we define

$$
z_{\lambda}=\prod_{i \geq 1} i^{m_{i}} m_{i} !
$$

We note that $z_{\lambda}$ is the order of the centralizer of an element of cycle-type $\lambda$ in $S_{n}$.

For a finite set $X$ and $\rho=(\rho(x))_{x \in X}$ a family of partitions indexed by $X$, we write

$$
\|\rho\|=\sum_{x \in X}|\rho(x)|
$$

It is convenient to regard $\rho=(\rho(x))_{x \in X}$ as a partition-valued function on $X$. We denote by $\mathcal{P}(X)$ the set of all partitions indexed by $X$ and by $\mathcal{P}_{n}(X)$ the set of all partitions in $\mathcal{P}(X)$ such that $\|\rho\|=n$. The total number of parts, denoted by $l(\rho)=\sum_{x} l(\rho(x))$, is called the length of $\rho$. Let $\mathcal{O P}(X)$ be the set of partitionvalued functions $(\rho(x))_{x \in X}$ in $\mathcal{P}(X)$ such that all parts of the partitions $\rho(x)$ are odd integers, and let $\mathcal{S P}(X)$ be the set of partition-valued functions $\rho \in \mathcal{P}(X)$ such that each partition $\rho(x)$ is strict. It is clear that $\left|\mathcal{O P}_{n}(X)\right|=\left|\mathcal{S P}_{n}(X)\right|$. When $X$ consists of a single element, we will omit $X$ and simply write $\mathcal{P}$ for $\mathcal{P}(X)$, thus $\mathcal{O P}$ or $\mathcal{S P}$ will be used similarly.

We denote by

$$
\begin{aligned}
& \mathcal{P}_{n}^{+}(X)=\left\{\lambda \in \mathcal{P}_{n}(X) \mid \quad l(\rho) \equiv 0 \bmod 2\right\}, \\
& \mathcal{P}_{n}^{-}(X)=\left\{\lambda \in \mathcal{P}_{n}(X) \mid \quad l(\rho) \equiv 1 \bmod 2\right\},
\end{aligned}
$$

and define $\mathcal{S P}_{n}^{ \pm}(X)=\mathcal{P}_{n}^{ \pm}(X) \cap \mathcal{S P}_{n}(X)$ for $i=0,1$.

The conjugacy classes of a wreath product is well understood, cf. $[\mathrm{M}]$. In particular this gives us the following description of conjugacy classes of the wreath product $H \Gamma_{n}=\left(\Gamma \times \mathbb{Z}_{2}\right)^{n} \rtimes S_{n}$.

Let $x=(g, \sigma)$ be an element in a conjugacy class of $\widetilde{H} \Gamma_{n}$, where $g=\left(g_{1}, \cdots, g_{n}\right)$ and $g_{i}=\left(\alpha_{i}, \varepsilon_{i}\right) \in \Gamma \times \mathbb{Z}_{2}$. We take the convention here that $\mathbb{Z}_{2}=\{ \pm 1\}$. For each cycle $y=\left(i_{1} i_{2} \cdots i_{k}\right)$ in the permutation $\sigma$ consider the element $\alpha_{y}=$ $\alpha_{i_{k}} \alpha_{i_{k-1}} \cdots \alpha_{i_{1}} \in \Gamma$ and $\varepsilon_{y}=\varepsilon_{i_{k}} \varepsilon_{i_{k-1}} \cdots \varepsilon_{i_{1}}$ (which is \pm 1 ) corresponding to the cycle $y$. For each $c \in \Gamma_{*}, \varepsilon= \pm$ and $r \geq 1$, let $m_{r}^{\varepsilon}(c)$ be the number of $r$-cycles in the 
permutation $\sigma$ such that the cycle product $\alpha_{y}$ lie in the conjugacy class $c$ and $\varepsilon_{y}$ equals $\varepsilon \cdot 1$. Then $c \rightarrow \rho_{\varepsilon}(c)=\left(1^{m_{1}^{\varepsilon}(c)} 2^{m_{2}^{\varepsilon}(c)} \ldots\right)$ defines a partition-valued function on $\Gamma_{*}$ for each $\varepsilon$. The partition-valued function $\rho_{\varepsilon}=\left(\rho_{\varepsilon}(c)\right)_{c \in \Gamma_{*}} \in \mathcal{P}\left(\Gamma_{*}\right)$ is called the $\varepsilon$-type of $x$. Denote by $\mathcal{P}\left(\Gamma_{*}\right)_{n}^{2}$ the set of pairs of partition-valued functions $\rho=\left(\rho^{+}, \rho^{-}\right)$such that $\left\|\rho^{+}\right\|+\left\|\rho^{-}\right\|=n$. The pair $\rho=\left(\rho^{+}, \rho^{-}\right)$is called the type of $x$. One can show that the type only depends on the conjugacy class of $x$ in $H \Gamma_{n}$ and the conjugacy classes in $H \Gamma_{n}$ are parameterized by the types $\rho \in \mathcal{P}\left(\Gamma_{*}\right)_{n}^{2}$. We will also say that the conjugacy class containing $x$ has conjugacy type $\rho$ and is denoted by $C_{\rho}$ if $x$ is of type $\rho$.

Denote by $D_{\rho}=\theta_{n}^{-1}\left(C_{\rho}\right)$. The following is established in [JW], Theorem 2.1.

Theorem 2.1 For $\rho=\left(\rho^{+}, \rho^{-}\right) \in \mathcal{P}\left(\Gamma_{*}\right)_{n}^{2}, D_{\rho}$ splits into two conjugacy classes in $\widetilde{H} \Gamma_{n}$ if and only if:

(1) for $D_{\rho}$ is even we have $\rho^{+} \in \mathcal{O P}_{n}\left(\Gamma_{*}\right)$ and $\rho^{-}=\emptyset$;

(2) for $D_{\rho}$ is odd we have $\rho^{+}=\emptyset$ and $\rho^{-} \in \mathcal{S P}_{n}^{-}\left(\Gamma_{*}\right)$.

Thus, the set $\left(H \Gamma_{n}\right)_{*}^{\text {e.s }}$ of even split conjugacy classes in $H \Gamma_{n}$ is in one-to-one correspondence with the set $\mathcal{O} \mathcal{P}_{n}\left(\Gamma_{*}\right)$.

\subsection{Spin supermodules over $\widetilde{H} \Gamma_{n}$}

The number of irreducible spin supermodules of $\widetilde{H} \Gamma_{n}$ equals the number of even split conjugacy classes in $\widetilde{H} \Gamma_{n}$, by a general theorem in the supermodule theory of finite supergroups [Jo1]. The next proposition follows from the equality $\left|\mathcal{S P}_{n}\left(\Gamma_{*}\right)\right|=\left|\mathcal{O P}_{n}\left(\Gamma_{*}\right)\right|$

Proposition 2.1 The number of irreducible spin supermodules of $\widetilde{H} \Gamma_{n}$ is equal to the number $\left|\mathcal{S P}_{n}\left(\Gamma_{*}\right)\right|$ of strict partition-valued functions on $\Gamma_{*}$.

We denote by $R^{-}\left(\widetilde{H} \Gamma_{n}\right)$ (resp. $\left.R_{\mathbb{Z}}^{-}\left(\widetilde{H} \Gamma_{n}\right)\right)$ the $\mathbb{C}$-span (resp. $\mathbb{Z}$-span) of the characters of irreducible spin supermodules of $\widetilde{H} \Gamma_{n}$. Let

$$
R_{\Gamma}^{-}=\bigoplus_{n=0}^{\infty} R^{-}\left(\widetilde{H} \Gamma_{n}\right) .
$$

When $\Gamma$ is trivial, we will simply drop the subscript $\Gamma$ and write

$$
R^{-}=\bigoplus_{n=0}^{\infty} R^{-}\left(\widetilde{H}_{n}\right)
$$

For example, when $\Gamma$ is trivial and thus $\widetilde{H} \Gamma_{n}$ reduces to $\widetilde{H}_{n}$, the irreducible spin supermodules of $\widetilde{H}_{n}$ are parameterized by strict partitions of $n$ (cf. [Ser] and [Jo2]). For strict partitions $\lambda$ and $\mu$ of $n$, let $T^{\lambda}$ and $T^{\mu}$ denote the corresponding irreducible spin supermodules over $\widetilde{H}_{n}$. We have 


$$
\operatorname{dim} \operatorname{Hom}_{\widetilde{H}_{n}}\left(T^{\lambda}, T^{\mu}\right)=\delta_{\lambda \mu} 2^{\delta(l(\lambda))}
$$

where the number $\delta(l(\lambda))$ is 0 for $l(\lambda)$ even and 1 otherwise. That is, the supermodule $T^{\lambda}$ is of type $M$ (resp. type $Q$ ) if and only if $l(\lambda)$ is even (resp. odd).

A most distinguished example of irreducible $\widetilde{H}_{n}$-supermodule is the so-called basic spin supermodule $L_{n}$ constructed as follows (cf. [Jo2, JW]). As a superspace $L_{n}$ is isomorphic to the group superalgebra $\mathbb{C}\left[\Pi_{n}\right] /\langle z=-1\rangle$ (i.e. the Clifford algebra in $n$ variables). Denote by $y_{i} \in L_{n}$ the image of $a_{i} \in \Pi_{n}$. Then $y_{I}=$ $\prod_{i \in I} y_{i}, I \subset\{1, \ldots, n\}$, form a linear basis of $L_{n}$. The action of $\widetilde{H}_{n}$ on $L_{n}$ is given by

$$
a_{j} y_{I}=y_{j} y_{I}(j=1, \ldots, n), \quad \sigma y_{I}=y_{s(I)}, s \in S_{n} .
$$

Indeed $L_{n}$ is exactly the $\widetilde{H}_{n}$-supermodule $T^{(n)}$ associated to the one-part partition $(n)$. If we denote by $\xi^{n}$ the character of $L_{n}$, then the character value of $\xi^{n}$ is $2^{l(\rho)}$ on a conjugacy class of type $\rho=\left(\rho^{+}, \emptyset\right)$, where $\rho^{+} \in \mathcal{O} \mathcal{P}_{n}\left(\Gamma_{*}\right)$.

For each partition-valued function $\rho=(\rho(c))_{c \in \Gamma_{*}}$ we define

$$
Z_{\rho}=2^{l(\rho)} \prod_{c \in \Gamma_{*}} z_{\rho(c)} \zeta_{c}^{l(\rho(c))},
$$

which is the order of the centralizer of an element in $H \Gamma_{n}$ of conjugacy type $\rho=$ $(\rho(c))_{c \in \Gamma_{*}}($ see $[\mathrm{JW}])$.

For a fixed $c \in \Gamma_{*}$, we denote by $c_{n}\left(n \in 2 \mathbb{Z}_{+}+1\right)$ the even split conjugacy class in $H \Gamma_{n}$ of the type $\left(\rho^{+}, \emptyset\right)$, where the partition-valued function $\rho^{+}$takes value the onepart partition $(n)$ at $c \in \Gamma_{*}$ and zero elsewhere. We denote by $\sigma_{n}(c) \in R^{-}\left(\widetilde{H} \Gamma_{n}\right)$ the class function of $\widetilde{H} \Gamma_{n}$ which takes value $n \zeta_{c}$ at the conjugacy class $c_{n}\left(c \in \Gamma_{*}\right)$ and zero otherwise. For $\rho=\left\{m_{r}(c)\right\}_{c, r} \in \mathcal{O} \mathcal{P}_{n}\left(\Gamma_{*}\right)$, we define

$$
\sigma^{\rho}=\prod_{c \in G_{*}, r \geq 1} \sigma_{r}(c)^{m_{r}(c)}
$$

and regard it as the class function on $\widetilde{H} \Gamma_{n}$ which takes value $Z_{\rho}$ at the conjugacy class $D_{\rho}^{+}$and 0 elsewhere. Then it follows that (cf. [Ser, Jo2])

$$
\xi^{n}=\sum_{\rho \in \mathcal{O P}_{n}\left(\Gamma_{*}\right)} 2^{l(\rho)} Z_{\rho}^{-1} \sigma^{\rho}
$$

Finally we define the analog for $\widetilde{H} \Gamma_{n}$ of Young subgroups of the symmetric groups. Let $\widetilde{H} \Gamma_{n} \tilde{\times} \widetilde{H} \Gamma_{m}$ be the direct product of $\widetilde{H} \Gamma_{n}$ and $\widetilde{H} \Gamma_{m}$ with a twisted multiplication

$$
\left(t, t^{\prime}\right) \cdot\left(s, s^{\prime}\right)=\left(t s z^{d\left(t^{\prime}\right) d(s)}, t^{\prime} s^{\prime}\right),
$$

where $s, t \in \widetilde{H} \Gamma_{n}, s^{\prime}, t^{\prime} \in \widetilde{H} \Gamma_{m}$. We define the spin product of $\widetilde{H} \Gamma_{n}$ and $\widetilde{H} \Gamma_{m}$ by letting

$$
\widetilde{H} \Gamma_{n} \hat{\times} \widetilde{H} \Gamma_{m}=\widetilde{H} \Gamma_{n} \tilde{\times} \widetilde{H} \Gamma_{m} /\{(1,1),(z, z)\}
$$


which carries a canonical $\mathbb{Z}_{2}$-grading and can be regarded as a subgroup of the supergroup $\widetilde{H} \Gamma_{n+m}$ in a natural way.

For two spin supermodules $U$ and $V$ of $\widetilde{H} \Gamma_{n}$ and $\widetilde{H} \Gamma_{m}$ we define a $\widetilde{H} \Gamma_{n} \hat{\times} \widetilde{H} \Gamma_{m}$ spin supermodule on the tensor product $U \otimes V$ by letting

$$
(t, s) \cdot(u \otimes v)=(-1)^{d(s) d(u)}(t u \otimes s v) .
$$

This induces an isomorphism $\phi_{n, m}$ from $R^{-}\left(\widetilde{H} \Gamma_{n}\right) \otimes R^{-}\left(\widetilde{H} \Gamma_{m}\right)$ to $R^{-}\left(\widetilde{H} \Gamma_{n} \hat{\times} \widetilde{H} \Gamma_{m}\right)$.

The space $R_{\Gamma}^{-}$carries a (commutative associative) multiplication which is defined by the composition (for all $n, m$ )

$$
R^{-}\left(\widetilde{H} \Gamma_{n}\right) \bigotimes R^{-}\left(\widetilde{H} \Gamma_{m}\right) \stackrel{\phi_{n, m}}{\longrightarrow} R^{-}\left(\widetilde{H} \Gamma_{n} \hat{\times} \widetilde{H} \Gamma_{m}\right) \stackrel{I n d}{\longrightarrow} R^{-}\left(\widetilde{H} \Gamma_{n+m}\right)
$$

\section{A decomposition theorem in equivariant $K$ - theory}

In this section we introduce a variant of equivariant $K$-theory for a finite supergroup. We recall a decomposition theorem in the equivariant $K$-theory from $[\mathrm{BC}, \mathrm{Ku}, \mathrm{AS}, \mathrm{HKR}]$, and present a generalization of it in our new setup.

\subsection{The standard version}

Given a (non-graded) finite group $\Gamma$ and a compact Hausdorff $\Gamma$-space $X$, we recall [Seg1] that $K_{\Gamma}^{0}(X)$ is the Grothendieck group of $G$-vector bundles over $X$. One can define $K_{\Gamma}^{1}(X)$ in terms of the $K^{0}$ functor and a certain suspension operation, and one puts

$$
K_{\Gamma}(X)=K_{\Gamma}^{0}(X) \bigoplus K_{\Gamma}^{1}(X) .
$$

In this paper we will be only concerned about $K_{\Gamma}(X) \otimes \mathbb{C}$, and subsequently we will denote

$$
\underline{K}_{\Gamma}(X)=K_{\Gamma}(X) \bigotimes \mathbb{C}
$$

We denote by $\operatorname{dim} K_{\Gamma}^{i}(X)(i=0,1)$ the dimension of $K_{\Gamma}^{i}(X) \otimes \mathbb{C}$.

If $X$ is locally compact, Hausdorff and paracompact $\Gamma$-space, take the one-point compactification $X^{+}$with the extra point $\infty$ fixed by $\Gamma$. Then we define $K_{\Gamma}^{0}(X)$ to be the kernel of the map

$$
K_{\Gamma}^{0}\left(X^{+}\right) \longrightarrow K_{\Gamma}^{0}(\{\infty\})
$$

induced by the inclusion map $\{\infty\} \hookrightarrow X^{+}$. This definition is equivalent to the earlier one when $X$ is compact. We also define $K_{\Gamma}^{1}(X)=K_{\Gamma}^{1}\left(X^{+}\right)$.

Note that $K_{\Gamma}(p t)$ is isomorphic to the Grothendieck ring $R_{\mathbb{Z}}(\Gamma)$ and $\underline{K}_{\Gamma}(p t)$ is isomorphic to the ring $R(\Gamma)$ of class functions on $\Gamma$. 
Let $X^{g}$ denote the fixed-point set by $g \in \Gamma$, which is preserved under the action of the centralizer $Z_{\Gamma}(g)$. The following decomposition theorem (cf. [BC, Ku, AS, HKR]) gives a description of each direct summand over conjugacy classes of $\Gamma$. We remark that the subspace of invariants $\underline{K}\left(X^{g}\right)^{Z_{\Gamma}(g)}$ is isomorphic to $\underline{K}\left(X^{g} / Z_{\Gamma}(g)\right)$, and it is isomorphic for different choice of $g$ in the same conjugacy class $[g] \in \Gamma_{*}$.

Theorem 3.1 There is a natural $\mathbb{Z}_{2}$-graded isomorphism

$$
\phi: \underline{K}_{\Gamma}(X) \longrightarrow \bigoplus_{[g] \in \Gamma_{*}} \underline{K}\left(X^{g}\right)^{Z_{\Gamma}(g)}
$$

\subsection{A super/twisted variant}

Now let $\widetilde{G}$ be a finite supergroup which contains a distinguished central element z of order 2 , and let $\theta$ be the quotient homomorphism $\widetilde{G} \rightarrow G=\widetilde{G} /\langle 1, z\rangle$.

Let $X$ be a compact (non-graded) $G$-space. We may regard $X$ as a $\widetilde{G}$-space by letting $g \in \widetilde{G}$ act by $\theta(g) \in G$. We denote by $\mathcal{C}_{\widetilde{G}}^{-}(X)$ the category whose objects consist of $\widetilde{G}$-equivariant complex vector superbundle (i.e. $\mathbb{Z}_{2}$-graded bundles) $E=$ $E_{0}+E_{1}$ (often denoted by $E_{0} \mid E_{1}$ ) over $X$ on which $\widetilde{G}$ acts in a $\mathbb{Z}_{2}$-graded manner and $z$ acts as -1 . We will call such an object a spin $\widetilde{G}$-vector superbundle. Given two objects $E, F$ in the category $\mathcal{C}_{\widetilde{G}}^{-}(X)$, the space of homomorphisms of $\widetilde{G}$-equivariant vector superbundles between $E$ and $F$ admits a natural $\mathbb{Z}_{2}$-grading:

$$
\operatorname{Hom}^{\widetilde{G}}(E, F)=\operatorname{Hom}_{0}^{\widetilde{G}}(E, F) \bigoplus \operatorname{Hom}_{1}^{\widetilde{G}}(E, F)
$$

By our definition of finite supergroups, $\widetilde{G}$ always contains odd elements. It follows that $\operatorname{rank} E_{0}=\operatorname{rank} E_{1}$ thanks to the existence of odd automorphisms from the odd elements of $\widetilde{G}$.

We denote by $K_{\widetilde{G}}^{-, 0}(X)$ the Grothendieck group of the abelian monoid of isomorphism classes ${ }^{2}$ of the vector superbundles in $\mathcal{C}_{\widetilde{G}}^{-}(X)$. As in the ordinary case, we can extend the definition of $\mathcal{C}_{\widetilde{G}}^{-}(X)$ to locally compact spaces, and define $K_{\widetilde{G}}^{-, 1}(X)$ to be $K_{\widetilde{G}}^{-, 0}(X \times \mathbb{R})$ where $\mathbb{R}$ is the real line. We denote $K_{\widetilde{G}}^{-}(X)=K_{\widetilde{G}}^{-, 0}(X)+K_{\widetilde{G}}^{-, 1}(X)$.

In this paper we will be only concerned about the free part $K_{\widetilde{G}}^{-}(X) \otimes \mathbb{C}$, which will be denoted by $\underline{K}_{\widetilde{G}}^{-}(X)$ subsequently. The following theorem, generalizing Theorem 3.1, is a variation of Adem-Ruan's construction [AR] for the so-called twisted equivariant $K$-theory. Recall that the character $\varepsilon_{g}$ of the centralizer group $Z_{G}(g)$ was defined in Lemma 2.1 and $Z_{G}(g)$ acts on $\underline{K}\left(X^{g}\right)$.

Theorem 3.2 Let $\widetilde{G}$ be a finite supergroup which contains a distinguished central element $z$ of order 2 , and let $\theta$ be the quotient homomorphism $\widetilde{G} \rightarrow G=\widetilde{G} /\langle 1, z\rangle$.

\footnotetext{
${ }^{2}$ The isomorphisms are $\mathbb{Z}_{2}$-graded and isomorphisms of degree 1 are allowed.
} 
Given a locally compact Hausdorff $G$-space $X$ and regarding it as a $\widetilde{G}$-space, we have a natural $\mathbb{Z}_{2}$-graded isomorphism

$$
\phi: \underline{K}_{\widetilde{G}}^{-}(X) \stackrel{\cong}{\longrightarrow} \bigoplus_{[g]}\left(\underline{K}\left(X^{g}\right) \bigotimes \varepsilon_{g}\right)^{Z_{G}(g)},
$$

where the summation runs over the even conjugacy classes in $G$.

Let us indicate briefly how the map $\phi$ is defined (also compare $[\mathrm{AR}]$ ). A $\widetilde{G}$ equivariant vector superbundle $E=E_{0} \mid E_{1}$, when restricted to $X^{g}$, becomes a spin $\widetilde{\langle g\rangle}$-vector superbundle, where $\widetilde{\langle g\rangle}$ denotes the subgroup of $\widetilde{G}$ covering the cyclic subgroup $\langle g\rangle$ of $G$ generated by $g$. Using Proposition 4.1, we can obtain the following isomorphisms of $Z_{G}(g)$-modules:

$$
K_{\widetilde{\langle g\rangle}}^{-}\left(X^{g}\right) \cong K\left(X^{g}\right) \bigotimes R^{-}(\widetilde{\langle g\rangle}) \cong K\left(X^{g}\right) \bigotimes R(\langle g\rangle) \bigotimes \varepsilon_{g}
$$

In this way we obtain a map $K_{\widetilde{G}}(X) \rightarrow K\left(X^{g}\right) \otimes R(\langle g\rangle) \otimes \varepsilon_{g}$. Composing this map with the character evaluation at $g$ gives us a map

$$
K_{\widetilde{G}}^{-}(X) \rightarrow K\left(X^{g}\right) \bigotimes \varepsilon_{g}
$$

whose image indeed is $Z_{G}(g)$-invariant.

Now we claim that this map is zero when $g$ is odd (thanks to the $\mathbb{Z}_{2}$-grading!) and thus the summation above does not involve the odd conjugacy classes. Let $g \in G$ be an odd element. Take an eigenvector $v_{0}+v_{1}$ of $g$ in the fiber of the ungraded subbundle $E^{\mu} \subset E_{0} \mid E_{1}$, where $v_{i} \in E_{i}(i=0,1)$. We see that $g \cdot v_{0}=\mu v_{1}$, and $g \cdot v_{1}=\mu v_{0}$. It follows that $v_{0}-v_{1}$ is an eigenvector of $g$ with eigenvalue $-\mu$, i.e. $v_{0}-v_{1} \in E^{-\mu}$. Denote by $\sharp$ the isomorphism of $E$ which is the identity map when restricted to $E_{0}$ and negative the identity map when restricted to $E_{1}$. Clearly $\sharp$ sends $E^{\mu}$ to $E^{-\mu}$ and vice versa. Thus the map (6) becomes zero since $\mu\left[E^{\mu}\right]+(-\mu)\left[E^{-\mu}\right]=0$. Putting (6) together for all even conjugacy classes, we obtain the map $\phi$.

The rest of the proof of the above theorem is the same as in $[A R]$ which in turn follows closely the classical case (cf. [BC, $\mathrm{Ku}, \mathrm{AS}]$ ).

Below we will single out a certain class of $\widetilde{G}$-space $X$ with a favorable property.

Definition 3.1 Assume we are in the setup of Theorem 3.2. We say the $\widetilde{G}$-space $X$ satisfies a strong vanishing property if for every even non-split conjugacy class $[g]$ in $G$, there exists some element $b$ in $Z_{G}(g)$ such that the character $\varepsilon_{g}$ of $Z_{G}(g)$ takes non-trivial value (which has to be -1) and the element $b$ fixes $X^{g}$ pointwise.

In view of Lemma 2.1 , if the $\widetilde{G}$-space $X$ satisfies the strong vanishing property, the isomorphism (5) will simplify to the following isomorphism 


$$
\phi: \underline{K}_{\widetilde{G}}^{-}(X) \stackrel{\cong}{\longrightarrow} \bigoplus_{[g]} \underline{K}\left(X^{g}\right)^{Z_{G}(g)},
$$

where the summation runs over all even split conjugacy classes in $G$.

When $X$ is a point the isomorphism $\phi$ becomes the map from a spin supermodule of $G$ to its character. As is known [Jo1, Jo2], the character of a spin supermodule vanishes on odd conjugacy classes as well on even non-split conjugacy classes. In our terminology, the one-point space for any $\widetilde{G}$ automatically satisfies the strong vanishing property. We shall see that the strong vanishing property holds for other non-trivial examples.

Remark 3.1 Theorem 3.2 contains Theorem 3.1 as a special case. Indeed, let $\widetilde{G}=\widetilde{H} \Gamma_{1}=\Pi_{1} \times \Gamma$ for some finite group $\Gamma$ and let $X$ be a $\Gamma$-space. We have an isomorphism

$$
K_{\Gamma}(X) \cong K_{\widetilde{H} \Gamma_{1}}^{\bar{n}}(X), \quad V \mapsto V \mid V
$$

On the other hand, we note that $\Pi_{1}$ is isomorphic to $\mathbb{Z}_{4}=\left\{1, a, z=a^{2}, a^{3}\right\}$ with the $\mathbb{Z}_{2}$-grading given by letting the generator $a$ be of degree 1 . The quotient of $\widetilde{G}$ by $\{1, z\}$ is $G=\Gamma \times \mathbb{Z}_{2}$. The even conjugacy class in $G$ is given by the conjugacy classes in $\Gamma \times\{1\}=\Gamma$. Therefore the right-hand side in Theorem 3.2 reduces to the right-hand side of Theorem 3.1.

It is possible to further generalize Theorem 3.2 along the line of $[\mathrm{AR}]$.

\section{The group $\widetilde{H}_{n}$ and $K$-theory operations}

In this section, we construct various $K$-theory operations based on the finite supergroup $\widetilde{H}_{n}$. This is an analog of Atiyah's construction [At] of $K$-theory operations by using the symmetric groups and implicitly Schur duality. The role of Schur duality is replaced here by the Sergeev's generalization [Ser] of the Schur duality involving $\widetilde{H}_{n}$.

\subsection{The group $\widetilde{H}_{n}$ and a ring of symmetric functions}

Let $V$ be a complex vector space of dimension $m$. We denote by $q(V)$ the superalgebra of linear transformations on the superspace $V \mid V=V+V$ which preserve an odd automorphism $P: V|V \rightarrow V| V$ such that $P^{2}=-1$. For example, if we take $V=\mathbb{C}^{m}$, and $P$ to be given by the $2 m \times 2 m$ matrix

$$
\left[\begin{array}{cc}
0 & I \\
-I & 0
\end{array}\right]
$$

then $q(V)$ is the Lie superalgebra which is obtained by the associative superalgebra $Q(m)$ (see Section 2) by taking the supercommutators. 
Let us now consider the natural action of $q(V)$ on $V \mid V$. We may form the $n$-fold tensor product $(V \mid V)^{\otimes n}$, on which $q(V)$ acts naturally. In addition we have an action of the finite supergroup $\widetilde{H}_{n}$ : the symmetric group $S_{n}$ acts on $(V \mid V)^{\otimes n}$ by permutations with appropriate signs; $a_{i}$ acts on $(V \mid V)^{\otimes n}$ by means of exchanging the parity of $i$-th copy of $V \mid V$ via the odd automorphism $P$ of $V \mid V$. More explicitly, $a_{i}$ transforms the vector $v_{1} \otimes \ldots v_{i-1} \otimes v_{i} \otimes \ldots \otimes v_{n}$ in $(V \mid V)^{\otimes n}$ into $(-1)^{p\left(v_{1}\right)+\ldots+p\left(v_{i-1}\right)} v_{1} \otimes \ldots v_{i-1} \otimes P\left(v_{i}\right) \otimes \ldots \otimes v_{n}$. According to Sergeev [Ser], the actions of $q(m)$ and $\widetilde{H}_{n}$ (super)commute with each other. Furthermore, one has

$$
(V \mid V)^{\otimes n} \cong \sum_{\lambda} 2^{-\delta(l(\lambda))} U_{m}^{\lambda} \otimes T^{\lambda}
$$

where $T^{\lambda}$ is the irreducible $\widetilde{H}_{n}$-supermodule associated to a strict partition $\lambda$, and $U_{m}^{\lambda}=\operatorname{Hom}_{\widetilde{H}_{n}}\left(T^{\lambda},(V \mid V)^{\otimes n}\right)$ is an irreducible $q(m)$-module. The expression $2^{-\delta(l(\lambda))} U_{m}^{\lambda} \otimes T^{\lambda}$ above has the following meaning. Suppose that $A$ and $B$ are two supergroups or two superalgebras and suppose that $V_{A}$ and $V_{B}$ are irreducible supermodules over $A$ and $B$ of type $Q$, namely, $\operatorname{Hom}_{A}\left(V_{A}, V_{A}\right)$ and $\operatorname{Hom}_{B}\left(V_{B}, V_{B}\right)$ are both isomorphic to the Clifford superalgebra in one odd variable. It is known (cf. e.g. [Jo1]) that $V_{A} \otimes V_{B}$ as a module over $A \otimes B$ is not irreducible, but decomposes into a direct sum of two isomorphic copies (via an odd isomorphism) of the same irreducible supermodule. In our particular setting when $l(\lambda)$ is odd both $T^{\lambda}$ and $U_{m}^{\lambda}$ are such modules (cf. [Ser]). So in this case we mean to take one copy inside their tensor product.

We introduce (cf. $[\mathrm{M}]$ ) the symmetric functions $q_{n}$ in the variables $x_{1}, x_{2}, \ldots$ by the formula

$$
\sum_{n \geq 0} q_{n} t^{n}=\prod_{i} \frac{1+x_{i} t}{1-x_{i} t} .
$$

Denote by $\Omega$ the subring of symmetric functions generated by $q_{1}, q_{2}, q_{3}, \cdots$, and $\Omega^{n}$ the subspace spanned by symmetric functions in $\Omega$ of degree $n$. Put $\Omega_{\mathbb{C}}=\Omega \otimes_{\mathbb{Z}} \mathbb{C}$ and $\Omega_{\mathbb{C}}^{n}=\Omega^{n} \otimes_{\mathbb{Z}} \mathbb{C}$. Recall $[\mathrm{M}]$ that a linear basis of $\Omega_{\mathbb{C}}$ is given a distinguished class of symmetric functions $Q_{\lambda}$, call the Schur $Q$-functions, parameterized by strict partitions $\lambda$. Furthermore $\Omega^{\mathbb{C}}=\mathbb{C}\left[q_{1}, q_{3}, q_{5}, \cdots\right]$ where $q_{r}(r$ odd $)$ are algebraically independent. We take the convention that when the set of variables is finite, say $x=\left(x_{1}, x_{2}, \ldots, x_{m}\right)$, we set $Q_{\lambda}(x)=Q_{\lambda}\left(x_{1}, x_{2}, \ldots, x_{m}, 0,0 \ldots\right)$. According to Sergeev $\left[\right.$ Ser], the trace of the diagonal matrix $D=\operatorname{diag}\left(x_{1}, \ldots, x_{m} ; x_{1}, \ldots, x_{m}\right)$ in $q(m)$ acting on $U_{m}^{\lambda}$ is

$$
\operatorname{Tr} D \mid U_{m}^{\lambda}=2^{\frac{\delta(l(\lambda))-l(\lambda)}{2}} Q_{\lambda}(x) .
$$

Given a spin $\widetilde{H}_{n}$-supermodule $W$, we define $W(V)$ to be the space of $\widetilde{H}_{n^{-}}$ invariants $\left(W \otimes(V \mid V)^{\otimes n}\right)^{\widetilde{H}_{n}}$. It is easy to check that the correspondence $V \mapsto$ $W(V)$ is functorial on $V$. In particular, if we take a diagonalizable linear transformation $l: V \rightarrow V$ with eigenvalues $x_{1}, \ldots, x_{m}$, then the eigenvalues of the induced 
map $W(l)=I d_{W} \otimes(l \mid l)^{\otimes n}: W(V) \rightarrow W(V)$ are monomials in $x_{1}, \ldots, x_{m}$ of degree $n$. In particular the trace of $W(l)$ is a symmetric polynomial in $x_{1}, \ldots, x_{m}$ of degree $n$ with integer coefficients. One can argue that this symmetric polynomial (for $m \geq n)$ is the restriction of a unique symmetric function in infinite many variables.

By definition we have $\left(W_{1} \oplus W_{2}\right)(V)=W_{1}(V) \oplus W_{2}(V)$. It follows the mapping $W \mapsto \operatorname{Tr} W(l)$ induces a map, denoted by $c h$, from $R^{-}\left(\widetilde{H}_{n}\right)$ to the space of symmetric functions of degree $n$. Note that $W(V) \cong \operatorname{Hom}_{\widetilde{H}_{n}}\left(W,(V \mid V)^{\otimes n}\right)$ since all the character values of $W$ are real, i.e. $W$ is self-dual. It follows from (7) that if we take $W$ to be $T^{\lambda}$ then $c h$ sends the class function associated with $T^{\lambda}$ to $2^{\frac{\delta(l(\lambda))-l(\lambda)}{2}} Q_{\lambda}(x)$. In this way we have defined a map ch from $R^{-}=\oplus_{n} R^{-}\left(\widetilde{H}_{n}\right)$ to the ring $\Lambda_{\mathbb{C}}$ of symmetric functions.

Remark 4.1 It is possible that one can develop this approach coherently to study the map $c h: R^{-} \rightarrow \Lambda_{\mathbb{C}}$ without referring to the Lie superalgebra $q(V)$ and thus essentially independent of the work of Sergeev [Ser], as sketched below. This is a super analog of an approach adopted in Knutson $[\mathrm{Kn}]$ in the setup of symmetric groups. For example, we can start by arguing that the space $W(V)$ associated to the basic spin supermodule $L_{n}$ (with character $\xi_{n}$ ) is the $n$-th supersymmetric algebra $Q^{n}(V \mid V)=\oplus_{i=0}^{n} S^{i} V \otimes \Lambda^{n-i} V$, and thus $\operatorname{ch}\left(\xi^{n}\right)=\sum_{i=0}^{n} h_{i} e_{n-i}=q_{n}(\mathrm{cf}$. Ex. 6(c), pp261, [M]), which is exactly the Schur $Q$-function $Q_{(n)}$. Here $h_{r}$ and $e_{r}$, which stand for the $r$-th elementary and complete symmetric function respectively, are the traces of $\operatorname{diag}\left(x_{1}, \ldots, x_{m}\right)$ on $S^{r} V$ and $\Lambda^{r} V$. One can easily show by using the Frobenius reciprocity that $c h: R^{-}\left(\widetilde{H}_{n}\right) \rightarrow \Lambda$ is an algebra homomorphism. The image of $c h$ contains $\Omega_{\mathbb{C}}^{n}$ as we have just seen that it contains $q_{n}$ for all $n$. By comparing the dimensions, the characteristic map $c h: R^{-} \rightarrow \Omega_{\mathbb{C}}$ is indeed an isomorphism.

There is another way to define the characteristic map as follows (cf. [Jo2, Ser]). Denote by $p_{n}$ the $n$-th power sum symmetric functions, and define $p_{\mu}=$ $p_{\mu_{1}} p_{\mu_{2}} \ldots$ for a partition $\mu=\left(\mu_{1}, \mu_{2}, \ldots\right)$. Given a character $\chi \in R^{-}$, we define the characteristic map $c h^{\prime}: R^{-} \rightarrow \Omega_{\mathbb{C}}$ by

$$
\operatorname{ch}^{\prime}(\chi)=\sum_{\mu \in \mathcal{O} \mathcal{P}_{n}} z_{\mu}^{-1} \chi_{\mu} p_{\mu}
$$

where $z_{\mu}=\prod_{i>1} i^{m_{i}} m_{i}$ ! for $\mu=\left(1^{m_{1}} 3^{m_{3}} \ldots\right)$ and $\chi_{\mu}$ the character value at the even split conjugacy class $D_{\mu, \emptyset}^{+}$. It is known [Jo2] that $c h^{\prime}$ is an algebra isomorphism and it sends $\xi^{n}$ to $q_{n}$ for all $n$. Thus $c h^{\prime}$ coincides with the characteristic map $c h$ we defined above.

\section{$4.2 \quad$ K-theory operations}

Let $\widetilde{G}$ is a finite supergroup with $G=\widetilde{G} /\langle 1, z\rangle$, and let $X$ be a trivial $G$-space (and thus $\widetilde{G}$-space). Given a $G$-supermodule $V$ in $R_{\mathbb{Z}}^{-}(G)$ and a complex vector 
bundle $E$ on $X, E \otimes V$ can be given a natural $\widetilde{G}$-equivariant vector superbundle structure over $X$ by letting $\widetilde{G}$ act on only the factor $V$. Obviously $E \otimes V$ lies in the category $\mathcal{C}_{\widetilde{G}}(X)$. In this way we obtain a map $R_{\mathbb{Z}}^{-}(\widetilde{G}) \otimes K(X) \longrightarrow K_{\widetilde{G}}^{-}(X)$. Then we have the following (compare [Seg1]).

Proposition 4.1 Under the above setup, there is a canonical isomorphism

$$
K_{\widetilde{G}}^{-}(X) \stackrel{\cong}{\longrightarrow} K(X) \bigotimes R_{\mathbb{Z}}^{-}(\widetilde{G}) .
$$

One needs some extra care to define the inverse of the isomorphism in the above proposition. Given a $G$-supermodule $V$ in $R_{\mathbb{Z}}^{-}(G)$ and a complex vector bundle $E$ on $X$, consider $\operatorname{Hom}_{G}(\mathbf{V}, E)$, where $\mathbf{V}$ is the trivial $G$-superbundle over $X$ associated to $V$. $\operatorname{Hom}_{G}(\mathbf{V}, E)$ is isomorphic to $E$ if $V$ is of type $M$, but isomorphic to $E \otimes C_{1}=E \mid E$ if $V$ is of type $Q$, where $C_{1}$ is the Clifford algebra in one variable. It is perhaps more natural to replace $K(X)$ in the proposition above by an isomorphic space $K_{\Pi_{1}}^{-}(X)$, cf. Remark 3.1 .

Below we will construct various $K$-theory operations based on the construction in the previous subsection. It is a super analog of an approach due to Atiyah [At] who used the symmetric group representations.

Let $E$ be a vector bundle over $X$, consider the $n$-th tensor power $(E \mid E)^{\otimes n}$ of the vector superbundle $E \mid E$. The odd operator $P\left(P^{2}=-1\right)$ acts on each factor $E \mid E$ fiberwise and induces an action of the finite supergroup $\Pi_{n}$ on $(E \mid E)^{\otimes n}$. The symmetric group $S_{n}$ also acts on $(E \mid E)^{\otimes n}$ in a natural way. The joint action of $\Pi_{n}$ and $S_{n}$ then gives rise to an action of the finite supergroup $\widetilde{H}_{n}$ on $(E \mid E)^{\otimes n}$. We have the following decomposition

$$
(E \mid E)^{\otimes n} \cong \sum_{\pi} 2^{-\delta(l(\pi))} T^{\pi} \bigotimes \pi(E)
$$

where $\pi$ is a strict partition of $n$, and $\pi(E)=\left(T^{\pi} \otimes(E \mid E)^{\otimes n}\right)^{\widetilde{H}_{n}}$ is a vector bundle on $X$. Clearly one can extend the definition of the vector bundle $\pi(E)$ associated to any spin supermodule $\pi$ of $\widetilde{H}_{n}$ so that it is additive on $\pi$. In this way we obtain a ring homomorphism $j^{-}: R^{-} \rightarrow \mathrm{Op}(K)$, where $R^{-}=\bigoplus_{n} R^{-}\left(\widetilde{H}_{n}\right)$ and $\operatorname{Op}(K)$ is the ring of $K$-theory operations on $K(X)$ (cf. [At]).

We note that if $\pi$ is the one-part partition $(n)$ then $\pi(E)$ is the $n$th supersymmetric power $Q^{n}$ :

$$
Q^{n}(E \mid E)=\sum_{i=0}^{n} S^{i} E \otimes \Lambda^{n-i} E
$$

For odd $n$, we denote by $\psi^{n}$ the operation corresponding to the class function $\sigma_{n} \in R^{-}\left(\widetilde{H}_{n}\right)$ which takes value $n$ in the even split conjugacy class of type $((n), \emptyset)$ and zero elsewhere. Denote by $Q_{t}(E)=\sum_{n=0}^{\infty} Q^{n}(E \mid E) t^{n}$, where $t$ is a formal variable. 
Proposition 4.2 The operation $\psi^{r}$ (r odd) coincides with the usual rth Adams operation. In particular it is additive. Furthermore, we have

$$
Q_{t}(E)=\exp \left(\sum_{r>0, \text { odd }} 2 \psi^{r}(E) t^{r} / r\right) .
$$

Proof. We first prove (9). Since the operations $Q^{n}$ 's and $\psi^{r}$ 's are obtained from the ring homomorphism $j^{-}: R^{-} \rightarrow \mathrm{Op}(K)$, we only need to show that the corresponding identity holds in $R^{-}$, or alternatively in $\Omega_{\mathbb{C}}$. But this is a classical identity $[\mathrm{M}]$

$$
\sum_{n \geq 0} q_{n} t^{n}=\exp \left(\sum_{r>0, o d d} 2 p_{r} t^{r} / r\right)
$$

Now we denote by $\sigma_{t}=\sum_{n} \sigma^{n} t^{n}, \Lambda_{t}=\sum_{n} \Lambda^{n} t^{n}$. Let us denote by $\tilde{\psi}^{r}$ the $r$ th Adams operations for the time being. It is classical that

$$
\begin{aligned}
& \sigma_{t}(E)=\exp \left(\sum_{r>0} \tilde{\psi}^{r}(E) t^{r} / r\right) \\
& \Lambda_{t}(E)=\exp \left(\sum_{r>0}(-1)^{r-1} \tilde{\psi}^{r}(E) t^{r} / r\right) .
\end{aligned}
$$

We have from (8) that $Q_{t}(E)=\Lambda_{t}(E) \sigma_{t}(E)$ and therefore

$$
Q_{t}(E)=\exp \left(\sum_{r>0, o d d} 2 \tilde{\psi}^{r}(E) t^{r} / r\right)
$$

Comparing with (9) which we have already established, we have $\psi^{r}=\tilde{\psi}^{r}$.

It follows that $Q_{t}(E+F)=Q_{t}(E) Q_{t}(F)$ and $Q_{t}(E-F)=Q_{t}(E) Q_{-t}(F)$, where $E, F \in K(X)$, since the Admas operations are additive. For example, the second equation reads componentwise as follows:

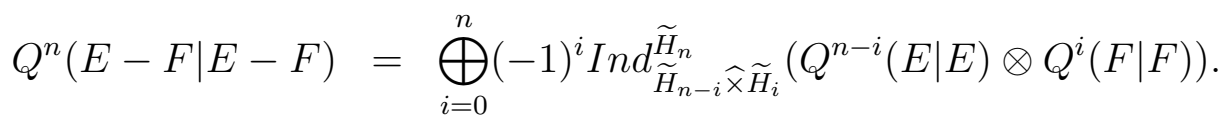

Remark 4.2 The ring $\Lambda$ of symmetric functions is a basic model for (free) $\lambda$ rings, and indeed $\lambda$ rings can be defined by axiomizing various properties of natural operations on $\Lambda$, where the Adams operations play a crucial role (cf. $[\mathrm{Kn}]$ ). We can define similarly a notion of $Q-\lambda$ ring with Adams operations of odd degrees only, using $\Omega_{\mathbb{C}}$ as a basic model. Then what we have just shown is that $K(X)$ admits a $Q-\lambda$ ring structure. We will see the structure of a $Q-\lambda$ ring instead of a $\lambda$ ring shows up naturally in some fairly non-trivial setup in Section 5. 


\section{$5 \quad$ Algebraic structures on $\mathcal{F}_{\Gamma}^{-}(X)$}

In this section, we shall study in detail the $K$-groups $\underline{K}_{\widetilde{H} \Gamma_{n}}^{-}\left(X^{n}\right)$ of generalized symmetric products for all $n$ simultaneously.

\subsection{Generalized symmetric products}

Our main examples in this paper are as follows. Let $\Gamma$ be a finite group and let $X$ be a $\Gamma$-space. The $n$-th Cartesian product $X^{n}$ is acted by the finite supergroup $\widetilde{H} \Gamma_{n}=\Pi_{n} \rtimes \Gamma_{n}$ in a canonical way: $\Pi_{n}$ acts trivially on $X^{n} ; \Gamma^{n}$ acts on $X^{n}$ factorwise while $S_{n}$ by permutations, and this gives rise to a natural action of the wreath product $\Gamma_{n}=\Gamma^{n} \rtimes S_{n}$ by letting

$$
a .\left(x_{1}, \ldots, x_{n}\right)=\left(g_{1} x_{s^{-1}(1)}, \ldots, g_{n} x_{s^{-1}(n)}\right)
$$

where $a=\left(\left(g_{1}, \ldots, g_{n}\right), s\right) \in \Gamma_{n}$, and $x_{1}, \ldots, x_{n} \in X$. Note that orbifolds $X^{n} / S_{n}$ are often called symmetric products. We may refer to $X^{n} / \Gamma_{n}$, or $X^{n}$ with $\Gamma_{n^{-}}$ action, or rather $X^{n}$ with $\widetilde{H} \Gamma_{n}$-action as generalized symmetric products.

Our earlier general construction when applied to the generalized symmetric products gives us the category $\mathcal{C}_{\widetilde{H} \Gamma_{n}}^{-}\left(X^{n}\right)$ and its associated $K$-group $\underline{K}_{\widetilde{H} \Gamma_{n}}^{-}\left(X^{n}\right)$. It turns out this category affords an equivalent description below which affords more transparent geometric meaning.

The wreath product $\Gamma_{n}$ acts on the vector space $\mathbb{C}^{n}$ naturally by letting $\Gamma$ act trivially and $S_{n}$ act as the permutation representation. This action preserves the standard quadratic form on $\mathbb{C}^{n}$. We denote by $\mathbf{n}$ such a $\Gamma_{n}$-vector bundle $X^{n} \times \mathbb{C}^{n}$ over $X^{n}$. We denote by $C_{n}$ the complex Clifford algebra associated to $\mathbb{C}^{n}$ and the standard quadratic form on it. The action of $\Gamma_{n}$ on $\mathbb{C}^{n}$ induces a natural action on $C_{n}$. We denote by $C(\mathbf{n})$ the associated $\Gamma_{n}$-vector (super)bundle $X^{n} \times C_{n}$ on $X^{n}$, which is the Clifford module on $X^{n}$ associated to the vector bundle $\mathbf{n}$.

We introduce the following category $\mathcal{C}_{\Gamma_{n}}^{\mathbf{n}}\left(X^{n}\right)$ : the objects consist of complex vector superbundles $E=E_{0}+E_{1}$ on $X^{n}$ equipped with compatible actions of $\Gamma_{n}$ and the Clifford algebra $C_{n}$ associated to $\mathbb{C}^{n}$ with the standard quadratic form. That is, $E$ is a $\mathbb{Z}_{2}$-graded $C(\mathbf{n})$-module and a $G$-equivariant vector bundle over $X^{n}$ such that

$$
g \cdot(v \cdot \xi)=(g \cdot v) \cdot(g \cdot \xi), \quad g \in \Gamma_{n}, v \in \mathbf{n}, \xi \in E .
$$

Given two superbundles $E, F$ in $\mathcal{C}_{G}^{\mathbf{n}}\left(X^{n}\right)$, the space of $(G, C(\mathbf{n}))$-equivariant homomorphisms of vector superbundles admits a natural $\mathbb{Z}_{2}$-gradation. Since the twisted group algebra of $\Pi_{n}$ is isomorphic to the Clifford algebra $C_{n}$, the category $\mathcal{C}_{G}^{\mathbf{n}}(Y)$ is then obviously equivalent to the category $\mathcal{C}_{\widetilde{H} \Gamma_{n}}^{-}\left(X^{n}\right)$, and so are the corresponding $K$-groups.

When $X$ is a point, $K_{\widetilde{H} \Gamma_{n}}^{-}(p t)=K_{\widetilde{H} \Gamma_{n}}^{-, 0}(p t)$ reduces to the Grothendieck group $R_{\mathbb{Z}}^{-}\left(\widetilde{H} \Gamma_{n}\right)$ of the spin supermodules of $\widetilde{H} \Gamma_{n}$. 
Remark 5.1 We may replace the rank $n$ vector bundle $\mathbf{n}$ over $X^{n}$ above by the $n$th direct sum of a non-trivial line bundle endowed with a quadratic form, and modify the construction of the category $\mathcal{C}_{\Gamma_{n}}^{\mathbf{n}}\left(X^{n}\right)$ accordingly. We conjecture that the resulting $K$-group is isomorphic to $K_{\widetilde{H} \Gamma_{n}}^{\bar{n}}\left(X^{n}\right)$.

Remark 5.2 We may reverse the above consideration in a more general setup as below. Take a $G$-space $Y$ and a $G$-superbundle $C(\mathbf{n})$ over $Y$ whose fiber is the Clifford algebra in $n$ variables. Assume that there exists $n$ sections $a_{1}, \ldots, a_{n}$ of the superbundle $C(\mathbf{n})$ which fiberwise generate the Clifford algebra and $G$ permutes $a_{1}, \ldots, a_{n}$. It is of interest in algebraic topology (cf. Karoubi [Ka]) to study the category $\mathcal{C}_{G}^{\mathbf{n}}(Y)$ of $G$-equivariant vector superbundles over $Y$ which are compatible with the the Clifford module structure in the sense of (10) and study its associated $K$-group. Then we may form the finite supergroup $\Pi_{n} \rtimes G$ and reinterpret the category $\mathcal{C}_{G}^{\mathbf{n}}(Y)$ as the category $\mathcal{C}_{\Pi_{n} \rtimes G}^{-}(Y)$, and then apply Theorem 3.2 to the study of the associated $K$-group. In particular if we take $Y=X^{n}$ and $G=\Gamma_{n}$ for a $\Gamma$-space $X$, we recover our main examples of generalized symmetric products.

\subsection{Hopf algebra $\mathcal{F}_{\Gamma}^{-}(X)$}

Let $\widetilde{H}$ be a $\mathbb{Z}_{2}$-graded subgroup of a finite supergroup $\widetilde{G}$ with the same distinguished even element $z$, and let $X$ be a $G$-space $X$ which is regarded as a $\widetilde{G}$-space, where $G=\widetilde{G} /\langle 1, z\rangle$. we can define the restriction map $\operatorname{Res}_{H}^{G}: \underline{K}_{G}^{-}(X) \longrightarrow \underline{K}_{H}^{-}(X)$ and the induction map $\operatorname{Ind}_{H}^{G}: \underline{K}_{H}^{-}(X) \longrightarrow \underline{K}_{G}^{-}(X)$ in the same way as in the usual equivariant $K$-theory. When it is clear from the text, we will often abbreviate $\operatorname{Res}_{H}^{G}$ as $\operatorname{Res}_{H}$ or Res. Similar remarks apply to the induction map.

Now we introduce the direct sum of equivariant $K$-groups

$$
\mathcal{F}_{\Gamma}^{-}(X)=\bigoplus_{n \geq 0} \underline{K}_{\widetilde{H} \Gamma_{n}}^{-}\left(X^{n}\right), \quad \mathcal{F}_{\Gamma}^{-}(X, t)=\bigoplus_{n \geq 0} t^{n} \underline{K}_{\widetilde{H} \Gamma_{n}}^{\bar{z}}\left(X^{n}\right),
$$

where $\widetilde{H} \Gamma_{0}$ is the one-element group by convention, and $t$ is a formal variable counting the graded structure of $\mathcal{F}_{\Gamma}^{-}(X)$. We also set

$$
\operatorname{dim}_{t} \mathcal{F}_{\Gamma}^{-}(X)=\sum_{n \geq 0} t^{n} \operatorname{dim} \underline{K}_{\widetilde{H} \Gamma_{n}}^{\overline{(}}\left(X^{n}\right) .
$$

We define a multiplication $\cdot$ on the space $\mathcal{F}_{\Gamma}^{-}(X)$ by a composition of the induction map and the Künneth isomorphism $k$ :

$$
\underline{K}_{\widetilde{H} \Gamma_{n}}^{\bar{\tau}}\left(X^{n}\right) \otimes \underline{K}_{\widetilde{H} \Gamma_{m}}^{\overline{\widetilde{n}}}\left(X^{m}\right) \stackrel{k}{\longrightarrow} \underline{K}_{\widetilde{H} \Gamma_{n} \widehat{\times} \widetilde{H} \Gamma_{m}}^{-}\left(X^{n+m}\right) \stackrel{\text { Ind }}{\longrightarrow} \underline{K}_{\widetilde{H} \Gamma_{n+m}}^{-}\left(X^{n+m}\right) .
$$

We denote by 1 the unit in $\underline{K}_{\widetilde{H} \Gamma_{0}}^{-}\left(X^{0}\right)$ which can be identified with $\mathbb{C}$. 
On the other hand we can define a comultiplication $\Delta$ on $\mathcal{F}_{\Gamma}^{-}(X)$ to be a composition of the inverse of the Künneth isomorphism and the restriction from $\widetilde{H} \Gamma_{n}$ to $\widetilde{H} \Gamma_{m} \widehat{\times} \widetilde{H} \Gamma_{n-m}$ :

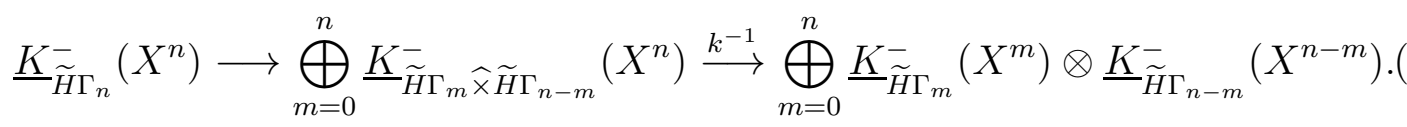

We define the counit $\epsilon: \mathcal{F}_{\Gamma}^{-}(X) \longrightarrow \mathbb{C}$ by sending $\underline{K}_{\widetilde{H} \Gamma_{n}}^{-}\left(X^{n}\right)(n>0)$ to 0 and $1 \in K_{\widetilde{H} \Gamma_{0}}^{-}\left(X^{0}\right)=\mathbb{C}$ to 1 .

Theorem 5.1 With various operations defined as above, $\mathcal{F}_{\Gamma}^{-}(X)$ is a graded Hopf algebra.

The proof is the same as the proof of the Hopf algebra structure on a direct sum of equivariant $K$-groups $\oplus_{n} \underline{K}_{\Gamma_{n}}\left(X^{n}\right)$ [W1], where a straightforward generalization to the equivariant $K$-groups of the Mackey's theorem plays a key role. One easily checks the super version of the Mackey's theorem can be carried over to our $K$ group setup. In the case when $X$ is a point and thus $\underline{K}_{\widetilde{H} \Gamma_{n}}^{-}\left(X^{n}\right) \cong R^{-}\left(\widetilde{H} \Gamma_{n}\right)$, the Hopf algebra structure above is also treated in $[\mathrm{JW}]$.

\subsection{Description of the algebra $\mathcal{F}_{\Gamma}^{-}(X)$}

Take an even split element $a=(\gamma, \sigma)$ in $H \Gamma_{n}$ of type $\rho=\left(\rho^{+}, \emptyset\right)$, where $g=$ $\left(g_{1}, \cdots, g_{n}\right), g_{i}=\left(\alpha_{i}, \varepsilon_{i}\right) \in \Gamma \times \mathbb{Z}_{2}$, and $\rho^{+}=\left(m_{r}^{+}(c)\right)_{r, c} \in \mathcal{O} \mathcal{P}_{n}\left(\Gamma_{*}\right)$. We define $\bar{a}=\left(\left(\alpha_{1}, \cdots, \alpha_{n}\right), \sigma\right) \in \Gamma_{n}=\Gamma^{n} \rtimes S_{n}$. Since the subgroup $\Pi_{n} \leq H \Gamma_{n}$ acts on $X^{n}$ trivially, the orbit space $\left(X^{n}\right)^{a} / Z_{H \Gamma_{n}}(a)$ is identified with $\left(X^{n}\right)^{\bar{a}} / Z_{\Gamma_{n}}(\bar{a})$ which has been calculated earlier in Lemmas 4 and 5 of [W1]. We make a convention here to denote the centralizer $Z_{G}(g)$ (resp. $\left.X^{g}, X^{g} / Z_{G}(g)\right)$ by $Z_{G}(c)\left(\operatorname{resp} . X^{c}, X^{c} / Z_{G}(c)\right)$ by abuse of notations when the choice of a representative $g$ in a conjugacy class $c$ of a group $G$ is irrelevant. For a fixed $c \in \Gamma_{*}$, recall that $c_{n}\left(n \in 2 \mathbb{Z}_{+}+1\right)$ is the even split conjugacy class in $H \Gamma_{n}$ of the type $\left(\rho^{+}, \emptyset\right)$, where the partition-valued function $\rho^{+}$takes value the one-part partition $(n)$ at $c \in \Gamma_{*}$ and zero elsewhere.

Lemma 5.1 Let $a \in H \Gamma_{n}$ be an even split element of type $\rho=\left(\rho^{+}, \emptyset\right)$, where $\rho^{+}=\left(m_{r}^{+}(c)\right)_{r \geq 1, c \in \Gamma_{*}} \in \mathcal{O P}_{n}\left(\Gamma_{*}\right)$. Then the orbit space $\left(X^{n}\right)^{a} / Z_{H \Gamma_{n}}(a)$ can be naturally identified with

$$
\prod_{r, c} S^{m_{r}^{+}(c)}\left(X^{c} / Z_{\Gamma}(c)\right)
$$

where $S^{m}(\cdot)$ denotes the $m$-th symmetric product. In particular, the orbit space $\left(X^{n}\right)^{c_{n}} / Z_{H \Gamma_{n}}\left(c_{n}\right)$ can be naturally identified with $X^{c} / Z_{\Gamma}(c)$.

In view of Lemma 2.1, let us recall how we have classified the split conjugacy classes of $H \Gamma_{n}$ in Theorem 1.2 of [JW]. In order to show that a given element 
$a$ in $H \Gamma_{n}$ is non-split, an explicit element, say $b$, is constructed in the centralizer $Z_{H \Gamma_{n}}(a)$ such that the character $\varepsilon_{a}$ takes value -1 at $b$. This was achieved in [JW] case by case. On the other hand, we observe that in all cases the element $b$ fixes $\left(X^{n}\right)^{a}$ pointwise! In other words, we have the following.

Lemma 5.2 The $\widetilde{H} \Gamma_{n}$-space $X^{n}$ satisfies the strong vanishing property.

We now give an explicit description of $\mathcal{F}_{\Gamma}^{-}(X)=\bigoplus_{n \geq 0} \underline{K}_{\widetilde{H} \Gamma_{n}}^{-}\left(X^{n}\right)$ as a graded algebra.

Theorem 5.2 As a $\left(\mathbb{Z}_{+} \times \mathbb{Z}_{2}\right)$-graded algebra $\mathcal{F}_{\Gamma}^{-}(X, t)$ is isomorphic to the supersymmetric algebra $\mathcal{S}\left(\bigoplus_{r=1}^{\infty} t^{2 r-1} \underline{K}_{\Gamma}(X)\right)$. In particular, we have

$$
\operatorname{dim}_{t} \mathcal{F}_{\Gamma}^{-}(X)=\frac{\prod_{r=1}^{\infty}\left(1+t^{2 r-1}\right)^{\operatorname{dim} K_{\Gamma}^{1}(X)}}{\prod_{r=1}^{\infty}\left(1-t^{2 r-1}\right)^{\operatorname{dim} K_{\Gamma}^{0}(X)}} .
$$

Here the supersymmetric algebra is equal to the tensor product of the symmetric algebra $S\left(\bigoplus_{r=1}^{\infty} t^{2 r-1} \underline{K}_{\Gamma}^{0}(X)\right)$ and the exterior algebra $\Lambda\left(\bigoplus_{r=1}^{\infty} t^{2 r-1} \underline{K}_{\Gamma}^{1}(X)\right)$.

Proof. Take an even split element $a \in H \Gamma_{n}$ of type $\rho=\left(\rho^{+}, \emptyset\right)$, where $\rho^{+}=$ $\left(m_{r}^{+}(c)\right)_{r \geq 1, c \in \Gamma_{*}} \in \mathcal{P}_{n}\left(\Gamma_{*}\right)$. By Lemma 5.1 and the Künneth formula, we have

$$
\begin{aligned}
\underline{K}\left(\left(X^{n}\right)^{a} / Z_{H \Gamma_{n}}(a)\right) & \approx \bigotimes_{c \in \Gamma_{*}, r \geq 1 \text { odd }}\left(\left(\underline{K}\left(X^{c}\right)^{Z_{\Gamma}(c)}\right)^{\bigotimes m_{r}(c)}\right)^{S_{m_{r}^{+}(c)}} \\
& \approx \bigotimes_{c \in \Gamma_{*}, r \geq 1 \text { odd }} \mathcal{S}^{m_{r}^{+}(c)}\left(\underline{K}\left(X^{c} / Z_{\Gamma}(c)\right)\right) .
\end{aligned}
$$

We now calculate as follows. The statement concerning $\operatorname{dim}_{t} \mathcal{F}_{\Gamma}^{-}(X)$ follows from this immediately.

$$
\begin{aligned}
& \mathcal{F}_{\Gamma}^{-}(X, t) \cong \bigoplus_{n \geq 0} t^{n} \bigoplus_{[a] \in\left(H \Gamma_{n}\right)_{*}^{\text {e.s. }}} \underline{K}\left(\left(X^{n}\right)^{a} / Z_{H \Gamma_{n}}(a)\right) \\
& \text { by Theorem } 3.2 \text { and Lemma 5.2, } \\
& \cong \bigoplus_{n \geq 0} \bigoplus_{\left\{m_{r}^{+}(c)\right\}_{c, r} \in \mathcal{O P}_{n}\left(\Gamma_{*}\right)} t^{n} \bigotimes_{c, r \text { odd }} \mathcal{S}^{m_{r}^{+}(c)}\left(\underline{K}\left(X^{c} / Z_{\Gamma}(c)\right)\right) \\
& \text { by (13) and Theorem 2.1, } \\
& \cong \bigoplus_{\left\{m_{r}^{+}(c)\right\}_{c, r} \in \mathcal{O} \mathcal{P}\left(\Gamma_{*}\right)} \bigotimes_{c, r \text { odd }} \mathcal{S}^{m_{r}^{+}(c)}\left(t^{r} \underline{K}\left(X^{c} / Z_{\Gamma}(c)\right)\right) \\
& \cong \bigoplus_{\left\{m_{r}\right\}_{r}} \bigotimes_{r \geq 1 \text { odd }} \mathcal{S}^{m_{r}}\left(\bigoplus_{c \in \Gamma_{*}} t^{r} \underline{K}\left(X^{c} / Z_{\Gamma}(c)\right)\right) \quad \text { where } m_{r}=\sum_{c} m_{r}^{+}(c) \text {, } \\
& \cong \bigoplus_{\left\{m_{r}\right\}_{r} r \geq 1 \text { odd }} \bigotimes_{\mathcal{S}^{m_{r}}}\left(t^{r} \underline{K}_{\Gamma}(X)\right) \quad \text { by Theorem 3.1, } \\
& \cong \mathcal{S}\left(\bigoplus_{r=1}^{\infty} t^{2 r-1} \underline{K}_{\Gamma}(X)\right) \text {. }
\end{aligned}
$$


Recall that the orbifold Euler number $e(X, \Gamma)$ was introduced by Dixon, Harvey, Vafa and Witten [DHVW] in the study of orbifold string theory. It is subsequently interpreted as the Euler number of the equivariant $K$-group $K_{\Gamma}(X)$, cf. e.g. [AS]. If we define the Euler number of the generalized symmetric product to be the difference

$$
e\left(X^{n}, \widetilde{H} \Gamma_{n}\right):=\operatorname{dim} K_{\widetilde{H} \Gamma_{n}}^{-, 0}\left(X^{n}\right)-\operatorname{dim} K_{\widetilde{H} \Gamma_{n}}^{-, 1}\left(X^{n}\right),
$$

then we obtain the following corollary.

Corollary 5.1 The Euler number $e\left(X^{n}, \widetilde{H} \Gamma_{n}\right)$ is given by the following generating function:

$$
\sum_{n=0}^{\infty} e\left(X^{n}, \widetilde{H} \Gamma_{n}\right) t^{n}=\prod_{r=1}^{\infty}\left(1-t^{2 r-1}\right)^{-e(X, \Gamma)} .
$$

In the case when $X$ is a point, we obtain the following corollary (also cf. [JW]).

Corollary 5.2 When $X$ is a point and thus $\mathcal{F}_{\Gamma}^{-}(X) \cong R_{\Gamma}^{-}$, we have

$$
\sum_{n \geq 0} t^{n} \operatorname{dim} R^{-}\left(\widetilde{H} \Gamma_{n}\right)=\prod_{r=1}^{\infty}\left(1-t^{2 r-1}\right)^{-\left|\Gamma_{*}\right|}
$$

\subsection{Twisted vertex operators and $\mathcal{F}_{\Gamma}^{-}(X)$}

In the following, we will define various $K$-theory maps appearing in the following diagram $(n$ odd):

$$
\begin{aligned}
& \underline{K}_{\Gamma}(X) \stackrel{\aleph}{\longrightarrow} \underline{K}_{\widetilde{H} \Gamma_{1}}^{-}(X) \stackrel{\unrhd n}{\longrightarrow} \underline{K}_{\widetilde{H} \Gamma_{n}}^{-}\left(X^{n}\right) \\
& \stackrel{\phi_{n}}{\longrightarrow} \bigoplus_{[a] \in\left(H \Gamma_{n}\right)_{*}^{e . s}} \underline{K}\left(\left(X^{n}\right)^{a} / Z_{H \Gamma_{n}}(a)\right) \\
& \stackrel{p r}{\iota} \bigoplus_{c \in \Gamma_{*}} \underline{K}\left(\left(X^{n}\right)^{c_{n}} / Z_{H \Gamma_{n}}\left(c_{n}\right)\right) \\
& \stackrel{\vartheta}{\longrightarrow} \bigoplus_{c \in \Gamma_{*}} \underline{K}\left(X^{c} / Z_{\Gamma}(c)\right) \stackrel{\phi}{\longleftarrow} \underline{K}_{\Gamma}(X) .
\end{aligned}
$$

Noting that $\widetilde{H} \Gamma_{1}=\Gamma \times \Pi_{1}$, we have a canonical isomorphism, denoted by $\aleph$, from $K_{\Gamma}(X)$ to $K_{\widetilde{H} \Gamma_{1}}^{-}(X)$ given by $E \mapsto E \mid E$. Given a $\Gamma$-equivariant vector bundle $V$, consider the $n$-th outer tensor product $(V \mid V)^{\otimes n}$ which is a vector superbundle over $X^{n}$. The odd operator $P$ acting on each factor $V \mid V$ induces an action of the finite supergroup $\Pi_{n}$ on $(V \mid V)^{\bigotimes n}$ while the wreath product $\Gamma_{n}$ acts on $(V \mid V)^{\bigotimes n}$ by letting

$$
\left(\left(g_{1}, \ldots, g_{n}\right), s\right) \cdot u_{1} \otimes \ldots \otimes u_{n}=g_{1} u_{s^{-1}(1)} \otimes \ldots \otimes g_{n} u_{s^{-1}(n)}
$$


where $g_{1}, \ldots, g_{n} \in \Gamma, s \in S_{n}$ and $u_{i} \in V \mid V, i=1, \ldots, n$. It is easy to check the combined action gives rise to an action of the finite supergroup $\widetilde{H} \Gamma_{n}$ on $(V \mid V)^{\otimes n}$, and $(V \mid V)^{\otimes n}$ endowed with such an $\widetilde{H} \Gamma_{n}$ action is an $\widetilde{H} \Gamma_{n}$-equivariant vector superbundle over $X^{n}$. On the other hand we can define an $\widetilde{H} \Gamma_{n}$ action on $V^{\otimes n} \otimes\left(\mathbb{C}^{1 \mid 1}\right)^{\bigotimes n}$ as follows: $\Gamma_{n}$ acts on the first factor $V^{\bowtie n}$ only while $\Pi_{n}$ acts only on the second factor $\left(\mathbb{C}^{1 \mid 1}\right)^{\otimes n}$; the symmetric group $S_{n}$ acts diagonally. One can check that the combined action gives $V^{\bigotimes n} \otimes\left(\mathbb{C}^{1 \mid 1}\right)^{\bigotimes n}$ the structure of an $\widetilde{H} \Gamma$-equivariant vector superbundle over $X^{n}$. We easily see that $(V \mid V)^{\otimes n}$ is canonically isomorphic to $V^{\bowtie n} \otimes\left(\mathbb{C}^{1 \mid 1}\right)^{\bigotimes n}$ as a $\widetilde{H} \Gamma_{n}$-equivariant superbundle.

Remark 5.3 Note that the above $\left(\mathbb{C}^{1 \mid 1}\right)^{\bigotimes n}$ is precisely the basic $\widetilde{H} \Gamma_{n}$-supermodule $L_{n}$. In general for a given $\widetilde{H} \Gamma_{n}$-supermodule $M$ with character $\chi$, we can define an $\widetilde{H} \Gamma_{n}$-equivariant superbundle structure on $V^{\otimes n} \otimes M$ when replacing $L_{n}$ above by $M$. We will write the corresponding element in $K_{\widetilde{H} \Gamma_{n}}\left(X^{n}\right)$ as $V^{\otimes n} \star \chi$. This defines an additive map from $R\left(\Gamma_{n}\right)$ to $K_{\widetilde{H} \Gamma_{n}}\left(X^{n}\right)$ by sending $\chi$ to $V^{\bowtie n} \star \chi$.

Sending $V \mid V$ to $(V \mid V)^{\bigotimes n}$ gives rise to the K-theory map $\otimes n$. More explicitly, given $V, W$ two $\Gamma$-equivariant vector bundles on $X$, we use $V$ itself to denote the corresponding element in $K_{\Gamma}(X)$ by abuse of notation. Then

$$
(V|V-W| W)^{\bigotimes n}=\sum_{j=0}^{n}(-1)^{j} \operatorname{Ind}_{\widetilde{H} \Gamma_{n-j} \widehat{\widetilde{x}} \widetilde{H} \Gamma_{j}}^{\widetilde{\widetilde{H}} \Gamma_{n}}\left((V \mid V)^{\bigotimes(n-j)} \otimes(W \mid W)^{\bigotimes j}\right) .
$$

Here $(V \mid V)^{\otimes(n-j)}$ and $(W \mid W)^{\otimes j}$ carry the standard actions of $\widetilde{H} \Gamma_{n-j}$ and respectively $\widetilde{H} \Gamma_{j}$. The map $\phi_{n}$ is the isomorphism in Theorem 3.2 given by the summation $\sum_{\rho^{+} \in \mathcal{O P}_{n}\left(\Gamma_{*}\right)}\left(\phi_{n}\right)_{\rho^{+}}$over the even split conjugacy classes of $H \Gamma_{n}$ of type $\left(\rho^{+}, \emptyset\right)$ when applying to the case $X^{n}$ with the action of $\widetilde{H} \Gamma_{n}$. The map $p r$ is the projection to the direct sum over the even split conjugacy classes $c_{n}$ of $H \Gamma_{n}$ while $\iota$ denotes the inclusion map. The map $\vartheta$ denotes the natural identification given by Lemma 5.1. Finally the last map $\phi$ is the isomorphism given in Theorem 3.1.

We introduce in addition the following $K$-theory operations.

Definition 5.1 For $n \in 2 \mathbb{Z}_{+}+1$, we define the following $K$-theory operations as composition maps:

$$
\begin{aligned}
\psi^{n} & :=n \phi^{-1} \circ \vartheta \circ p r \circ \phi_{n} \circ \nabla n \circ \aleph: \underline{K}_{\Gamma}(X) \longrightarrow \underline{K}_{\Gamma}(X), \\
\varphi^{n} & :=n \phi_{n}^{-1} \circ \iota \circ p r \circ \phi_{n} \circ \nabla n: \underline{K}_{\widetilde{H} \Gamma_{1}}^{-}(X) \longrightarrow \underline{K}_{\widetilde{H} \Gamma_{n}}^{-}\left(X^{n}\right), \\
c h_{n} & :=\phi^{-1} \circ \vartheta \circ p r \circ \phi_{n}: \underline{K}_{\widetilde{H} \Gamma_{n}}\left(X^{n}\right) \longrightarrow \underline{K}_{\Gamma}(X), \\
\varpi_{n} & :=\phi_{n}^{-1} \circ \iota \circ \vartheta^{-1} \circ \phi: \underline{K}_{\Gamma}(X) \longrightarrow \underline{K}_{\widetilde{H} \Gamma_{n}}\left(X^{n}\right) .
\end{aligned}
$$

Recall that the notation $\psi^{n}$ ( $n$ odd) was used in Section 4 to denote the $n$th Adams operation. We shall see the $\psi^{n}$ defined here for $\Gamma$ trivial coincides with the 
$n$th Adams operation tensored with $\mathbb{C}$. This is why we have chosen to use the same notation. We list some properties of these K-theory maps which follows directly from defintions.

Proposition 5.1 The following identities hold:

$$
c h_{n} \circ \varpi_{n}=I \underline{\underline{K}}_{\Gamma}(X), \quad \varpi_{n} \circ \psi^{n}=\varphi^{n} \circ \aleph, \quad c h_{n} \circ \varphi^{n} \circ \aleph=\psi^{n},
$$

where $I \underline{\underline{K}}_{\Gamma}(X)$ denotes the identity operator on $\underline{K}_{\Gamma}(X)$.

Lemma 5.3 Both $\psi^{n}$ and $\varphi^{n}$ ( $n$ odd) are additive K-theory maps. In particular, for $\Gamma$ trivial, the operation $\psi^{n}$ given in the definition 5.1 coincides with the $n$th Adams operations on $\underline{K}(X)$.

Proof. We sketch a proof. By definition $\varpi_{n}$ is additive and $\aleph$ is an isomorphism. Thanks to the equality $\varpi_{n} \circ \psi^{n}=\varphi^{n} \circ \aleph$ by Proposition 5.1, to show that $\varphi^{n}$ is additive, it suffices to check that $\psi^{n}$ is additive. This can be proved in a parallel way by using (16) as Atiyah [At] proves the additivity of the Adams operations defined in terms of symmetric groups.

Now we set $\Gamma=\{1\}$ and consider the diagonal embedding $\Delta_{n}: X \rightarrow X^{\Delta} \hookrightarrow$ $X^{n}$. Since $\widetilde{H}_{n}$ acts on $X^{\Delta}$ trivially, it follows by Proposition 4.1 that $\underline{K}_{\widetilde{H}_{n}}^{-}(X) \cong$ $\underline{K}(X) \otimes R^{-}\left(\widetilde{H}_{n}\right)$. We have the following commutative diagram ( $n$ odd):

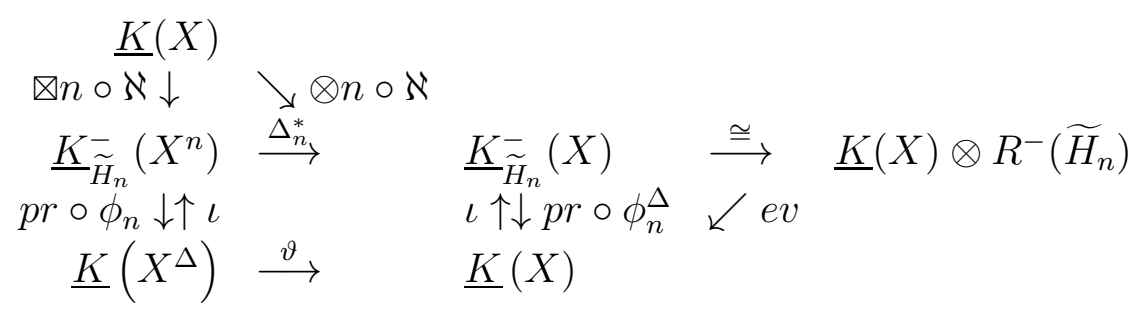

where $\phi_{n}^{\Delta}$ is the analog of $\phi_{n}$ when $X^{n}$ is replaced by the diagonal $X$, and the evaluation map $e v$ is defined to be the character value at the conjugacy class of type $((n), \emptyset)$. The map from $\underline{K}(X)$ to itself obtained along the left-bottom route in the above diagram coincides with $\frac{1}{n} \psi^{n}$ given in Definition 5.1. The map from $\underline{K}(X)$ to itself obtained along the top-right route in the above diagram gives $\frac{1}{n}$ times the $n$-th Adams operation. This of course gives another proof that both $\psi^{n}$ and $\varphi^{n}$ are additive when $\Gamma$ is trivial.

Remark 5.4 $\mathcal{F}_{\Gamma}^{-}(X)$ is a $Q-\lambda$ ring with $\varphi^{n}$ ( $n$ odd) as the $n$th Adams operation. If $X$ is a point and thus $\mathcal{F}_{\Gamma}^{-}(p t)=R_{\Gamma}^{-}$, then our result reduces to the fact that $\mathcal{F}_{\Gamma}^{-}(p t)$ is a free $Q-\lambda$ ring generated by $\Gamma_{*}$. In particular when $\Gamma$ is trivial this is isomorphic to the model $Q-\lambda$ ring $\Omega_{\mathbb{C}}$. 
Denote by $\widehat{\mathcal{F}}_{\Gamma}^{t}(X)$ the completion of $\mathcal{F}_{\Gamma}^{-}(X, t)$ which allows formal infinite sums. Given $V \in \underline{K}_{\Gamma}(X)$, we introduce $Q(V, t) \in \widehat{\mathcal{F}}_{\Gamma}^{t}(X)$ as follows:

$$
Q(V, t)=\bigoplus_{n \geq 0} t^{n}(V \mid V)^{\otimes n}
$$

The following lemma is immediate by Definition 5.1 and Remark 5.3.

Lemma 5.4 Given $V \in \underline{K}_{\Gamma}(X)$, we have (for $r$ odd)

$$
\varphi^{r} \circ \aleph(V)=\sum_{c \in \Gamma_{*}} \zeta_{c}^{-1} \phi_{r}^{-1}\left(\phi_{r}\right)_{c_{r}}\left(V^{\bigotimes r} \star \sigma_{r}(c)\right) .
$$

Proposition 5.2 Given $V \in \underline{K}_{\Gamma}(X)$, we can express $Q(V, t)$ as follows:

$$
Q(V, t)=\exp \left(\sum_{r>0 \text { odd }} \frac{2}{r} \varphi^{r} \circ \aleph(V) t^{r}\right) .
$$

Here the right-hand side is understood in terms of the algebra structure on $\mathcal{F}_{\Gamma}^{-}(X)$.

Proof. By (3) and the above lemma, we have

$$
\begin{aligned}
& Q(V, t)=\bigoplus_{n \geq 0} t^{n}\left(V^{\otimes n} \star \xi^{n}\right) \\
& =\bigoplus_{n \geq 0} t^{n}\left(\sum_{\rho \in \mathcal{O} \mathcal{P}_{n}\left(\Gamma_{*}\right)} \phi_{n}^{-1}\left(\phi_{n}\right)_{\rho}\left(V^{\otimes n} \star 2^{l(\rho)} Z_{\rho}^{-1} \sigma^{\rho}\right)\right) \\
& =\bigoplus_{n \geq 0} \sum_{\rho \in \mathcal{O P}_{n}\left(\Gamma_{*}\right)} \phi_{n}^{-1}\left(\phi_{n}\right)_{\rho}\left(2^{l(\rho)} Z_{\rho}^{-1} t^{n} V^{\bowtie n} \star \sigma^{\rho}\right) \\
& =\prod_{c \in G_{*}, r \geq 1 \text { odd }} \frac{1}{m_{r}(c) !}\left(\frac{2}{r} t^{r} \zeta_{c}^{-1} \phi_{r}^{-1}\left(\phi_{r}\right)_{c_{r}}\left(V^{\bigotimes r} \star \sigma_{r}(c)\right)\right)^{m_{r}(c)} \\
& =\exp \left(\sum_{r \geq 1 \text { odd }} \frac{2}{r} t^{r} \sum_{c \in \Gamma_{*}} \zeta_{c}^{-1} \phi_{r}^{-1}\left(\phi_{r}\right)_{c_{r}}\left(V^{凶 r} \star \sigma_{r}(c)\right)\right) \\
& =\exp \left(\sum_{r \geq 1 \text { odd }} \frac{2}{r} t^{r} \varphi^{r} \circ \aleph(V)\right) \text {. }
\end{aligned}
$$

Combining with the additivity of $\varphi^{r}$, the proposition implies

Corollary 5.3 The following equations hold for $V, W \in \underline{K}_{G}(X)$ :

$$
\begin{aligned}
Q(V \bigoplus W, t) & =Q(V, t) Q(W, t) \\
Q(-V, t) & =Q(V,-t) .
\end{aligned}
$$


Remark 5.5 The generating function $Q(V, t)$ is essentially half the twisted vertex operator, and the other half can be obtained by the adjoint operator to $Q(V, t)$. Twisted vertex operators have played an important role in the representation theory of infinite dimensional Lie algebras and the moonshine module, cf. [FLM]. When $X$ is a point, we can develop the picture more completely (cf. [JW]) to provide a group theoretic realization of vertex representations of twisted affine and twisted toroidal Lie algebras (also compare [FJW2] for a different construction).

\subsection{Twisted Heisenberg algebra and $\mathcal{F}_{\Gamma}^{-}(X)$}

We see from Theorem 5.2 that $\mathcal{F}_{\Gamma}^{-}(X)$ has the same size of the tensor product of the Fock space of an infinite-dimensional twisted Heisenberg algebra of rank $\operatorname{dim} K_{\Gamma}^{0}(X)$ and that of an infinite-dimensional twisted Clifford algebra of rank $\operatorname{dim} K_{G}^{1}(X)$. In this section we will actually construct such a Heisenberg/Clifford algebra, which we will simply refer to as a twisted Heisenberg (super)algebra from now on.

The dual of $\underline{K}_{\Gamma}(X)$, denoted by $\underline{K}_{\Gamma}(X)^{*}$, is naturally $\mathbb{Z}_{2}$-graded as identified with $\underline{K}_{\Gamma}^{0}(X)^{*} \oplus \underline{K}_{\Gamma}^{1}(X)^{*}$. Denote by $\langle\cdot, \cdot\rangle$ the pairing between $\underline{K}_{\Gamma}(X)^{*}$ and $\underline{K}_{\Gamma}(X)$. For any $m \in 2 \mathbb{Z}_{+}+1$ and $\eta \in \underline{K}_{\Gamma}(X)^{*}$, we define an additive map

$$
a_{-m}(\eta): \underline{K}_{G_{n}}\left(X^{n}\right) \longrightarrow \underline{K}_{G_{n-m}}\left(X^{n-m}\right)
$$

as the composition

$$
\begin{aligned}
\underline{K}_{\widetilde{H} \Gamma_{n}}^{-}\left(X^{n}\right) & \stackrel{\operatorname{Res}}{\longrightarrow} \underline{K}_{\widetilde{H} \Gamma_{m} \widehat{\times} \widetilde{H} \Gamma_{n-m}}\left(X^{n}\right) \stackrel{k^{-1}}{\longrightarrow} \underline{K}_{\widetilde{H} \Gamma_{m}}^{-}\left(X^{m}\right) \bigotimes \underline{K}_{\widetilde{H} \Gamma_{n-m}}^{-}\left(X^{n-m}\right) \\
& \stackrel{c h_{m} \otimes 1}{\longrightarrow} \underline{K}_{\Gamma}(X) \bigotimes \underline{K}_{\widetilde{H} \Gamma_{n-m}}\left(X^{n-m}\right) \stackrel{\eta \otimes 1}{\longrightarrow} \underline{K}_{\widetilde{H} \Gamma_{n-m}}^{-}\left(X^{n-m}\right) .
\end{aligned}
$$

On the other hand, we define for any $m \in 2 \mathbb{Z}_{+}+1$ and $V \in \underline{K}_{\Gamma}(X)$ an additive map

$$
a_{m}(V): \underline{K}_{\widetilde{H} \Gamma_{n-m}}^{-}\left(X^{n-m}\right) \longrightarrow \underline{K}_{\widetilde{H} \Gamma_{n}}^{-}\left(X^{n}\right)
$$

as the composition

$$
\begin{aligned}
\underline{K}_{\widetilde{H} \Gamma_{n-m}}^{-}\left(X^{n-m}\right) \stackrel{\frac{m}{2} \varpi_{m}(V) \otimes \cdot}{\longrightarrow} \underline{K}_{\widetilde{H} \Gamma_{m}}^{-}\left(X^{m}\right) \bigotimes \underline{K}_{\widetilde{H} \Gamma_{n-m}}^{-}\left(X^{n-m}\right) \\
\stackrel{k}{\longrightarrow} \quad \underline{K}_{\widetilde{H} \Gamma_{m} \widehat{\times} \widetilde{H} \Gamma_{n-m}}^{-}\left(X^{n}\right) \stackrel{\operatorname{Ind}}{\longrightarrow} \underline{K}_{\widetilde{H} \Gamma_{n}}^{-}\left(X^{n}\right) .
\end{aligned}
$$

Let $\mathcal{H}$ be the linear span of the operators $a_{-m}(\eta), a_{m}(V), m \in 2 \mathbb{Z}_{+}+1, \eta \in$ $\underline{K}_{\Gamma}(X)^{*}, V \in \underline{K}_{\Gamma}(X)$. Clearly $\mathcal{H}$ admits a natural $\mathbb{Z}_{2}$-gradation induced from that on $\underline{K}_{\Gamma}(X)$ and $\underline{K}_{\Gamma}(X)^{*}$. Below we shall use $[-,-]$ to denote the supercommutator as well. It is understood that $[a, b]$ is the anti-commutator $a b+b a$ when $a, b \in \mathcal{H}$ are both odd elements according to the $\mathbb{Z}_{2}$-gradation. 
Theorem 5.3 When acting on $\mathcal{F}_{\Gamma}^{-}(X), \mathcal{H}$ satisfies the twisted Heisenberg superalgebra commutation relations, namely for $m, l \in 2 \mathbb{Z}_{+}+1, \eta, \eta^{\prime} \in \underline{K}_{\Gamma}(X)^{*}$, $V, W \in \underline{K}_{\Gamma}(X)$, we have

$$
\begin{aligned}
{\left[a_{-m}(\eta), a_{l}(V)\right] } & =\frac{l}{2} \delta_{m, l}\langle\eta, V\rangle, \\
{\left[a_{m}(W), a_{l}(V)\right] } & =0 \\
{\left[a_{-m}(\eta), a_{-l}\left(\eta^{\prime}\right)\right] } & =0 .
\end{aligned}
$$

Furthermore, $\mathcal{F}_{\Gamma}^{-}(X)$ is an irreducible representation of the twisted Heisenberg superalgebra.

Remark 5.6 The proof of the Heisenberg algebra commutation relation can be given in a parallel way as the one for Theorem 4, [W1]. The irreducibility of $\mathcal{F}_{\Gamma}^{-}(X)$ as module over the Heisenberg algebra follows from Theorem 5.2. Given a bilinear form on $\underline{K}(X)$, then we can get rid of $\underline{K}(X)^{*}$ in the formulation of the above theorem. In the special case when $X$ is a point, the Heisenberg algebra here specializes to the one given in $[\mathrm{JW}]$ acting on $R_{\Gamma}^{-}$. In the case when $\Gamma$ is a finite subgroup of $S L_{2}(\mathbb{C})$, we may consider further the space which is the tensor product of $\mathcal{F}_{\Gamma}^{-}(p t)$ with a module of a certain 2-group which can be constructed out of $\Gamma$, and realize in this way a vertex representation of a twisted affine and a twisted toroidal Lie algebra. This is treated in [JW] in detail.

\section{Appendix: Another formulation using $\widetilde{S}_{n}$ and $\tilde{\Gamma}_{n}$}

As is well known (cf. e.g. [Sc, J, Jo, HH]), the symmetric group $S_{n}$ has a double cover $\widetilde{S}_{n}$ :

$$
1 \longrightarrow \mathbb{Z}_{2} \longrightarrow \widetilde{S}_{n} \stackrel{\theta_{n}}{\longrightarrow} S_{n} \longrightarrow 1
$$

generated by $z$ and $t_{i}, i=1, \cdots, n-1$ and subject to the relations:

$$
z^{2}=1, \quad t_{i}^{2}=\left(t_{i} t_{i+1}\right)^{3}=z, \quad t_{i} t_{j}=z t_{j} t_{i}(i>j+1), \quad z t_{i}=t_{i} z .
$$

The map $\theta_{n}$ sends $t_{i}$ 's to the simple reflections $s_{i}$ 's in $S_{n}$. The group $\widetilde{S}_{n}$ carries a natural $\mathbb{Z}_{2}$ grading by letting $t_{i}$ 's be odd and $z$ be even.

Given a finite group $\Gamma$, the symmetric group $S_{n}$ acts on the product group $\Gamma^{n}$, and the group $\widetilde{S}_{n}$ acts on $\Gamma^{n}$ via $\theta_{n}$. Thus we can form a semi-direct product $\widetilde{\Gamma}_{n}:=\Gamma^{n} \rtimes_{\theta_{n}} \widetilde{S}_{n}$, which carries a natural finite supergroup structure by letting elements in $\Gamma^{n}$ be even, cf. [FJW2]. We still denote by $\theta_{n}$ the quotient map $\widetilde{\Gamma}_{n} \rightarrow \widetilde{\Gamma}_{n} /\langle 1, z\rangle=\Gamma_{n}$. 
Given a $\Gamma$-space $X$, we have seen $X^{n}$ affords a natural $\Gamma_{n}$ action. Then we can apply the general construction in Sect. 3.2 to construct the category $\mathcal{C}_{\widetilde{\Gamma}_{n}}^{-}\left(X^{n}\right)$ and its associated $K$-group $K_{\widetilde{\Gamma}_{n}}^{-}\left(X^{n}\right)$. As before, we denote $\underline{K}_{\widetilde{\Gamma}_{n}}^{-}\left(X^{n}\right)=K_{\widetilde{\Gamma}_{n}}^{-}\left(X^{n}\right) \otimes \mathbb{C}$.

We then form the direct sum

$$
\begin{aligned}
\mathfrak{F}_{\Gamma}^{-}(X) & =\bigoplus_{n=0}^{\infty} \underline{K}_{\widetilde{\Gamma}_{n}}^{-}\left(X^{n}\right) \\
\mathfrak{F}_{\Gamma}^{-}(X, t) & =\bigoplus_{n=0}^{\infty} t^{n} \underline{K}_{\widetilde{\Gamma}_{n}}^{-}\left(X^{n}\right) .
\end{aligned}
$$

When $X$ is a point, $\underline{K}_{\widetilde{\Gamma}_{n}}^{-}(p t)$ reduces to the Grothendieck group $R^{-}\left(\widetilde{\Gamma}_{n}\right)$ of spin supermodules of $\widetilde{S}_{n}$, and $\mathfrak{F}_{\Gamma}^{-}(p t)$ has been studied in detail in [FJW2]. The purpose of the Appendix is to outline how to modify the various constructions of algebraic structures on $\mathcal{F}_{\Gamma}^{-}(X)$ for the new space $\mathfrak{F}_{\Gamma}^{-}(X)$. As the constructions are very similar to the $\mathcal{F}_{\Gamma}^{-}(X)$ case, we will be rather sketchy.

Given $n, m \geq 0$, we can define a $\mathbb{Z}_{2}$-graded subgroup $\widetilde{\Gamma}_{n} \widehat{\times} \widetilde{\Gamma}_{m}$ of $\widetilde{\Gamma}_{n+m}$, in a way analogous to (4). Then the obvious analog of constructions (11) and (12) defines a multiplication and comultiplication on the space $\mathfrak{F}_{\Gamma}^{-}(X)$. The following is an analog of Theorem 5.1 and it generalizes Theorem 3.8 of [FJW2] which is our special case when $X$ is a point.

Theorem 6.1 The space $\mathfrak{F}_{\Gamma}^{-}(X)$ carries a natural Hopf algebra structure.

By the analysis of the split conjugacy classes given in the proof of Theorem 2.5 of [FJW2], we see that the $\widetilde{\Gamma}_{n}$-space $X^{n}$ satisfies the strong vanishing property, and the analog of Lemma 5.1 holds. Therefore we obtain the following theorem which is an analog of Theorem 5.2.

Theorem 6.2 As a $\left(\mathbb{Z}_{+} \times \mathbb{Z}_{2}\right)$-graded algebra $\mathfrak{F}_{\Gamma}^{-}(X, t)$ is isomorphic to the supersymmetric algebra $\mathcal{S}\left(\oplus_{r=1}^{\infty} t^{2 r-1} \underline{K}_{\Gamma}(X)\right)$.

Except the first two terms and the first two arrows in the diagram (14), the rest of the diagram has a direct analog for $K_{\widetilde{\Gamma}_{n}}^{-}\left(X^{n}\right)$. Note in the definition of the $K$-theory maps $c h_{n}$ and $\varpi_{n}$ (see Definition 5.1) only the part of the diagram (14) which can be directly generalized to the $K_{\widetilde{\Gamma}_{n}}^{-}\left(X^{n}\right)$ setup has been used. Therefore analog of $c h_{n}$ and $\varpi_{n}$ can be defined in our new setup. This guarantees the analog of annihilation operators (18) and the creation operators (19) can be defined in our new setup. In this way we obtain the following which is an analog of Theorem 5.3.

Theorem 6.3 The space $\mathfrak{F}_{\Gamma}^{-}(X)$ affords an action of the twisted Heisenberg algebra $\mathcal{H}$ in terms of natural additive $K$-theory maps. Furthermore this representation is irreducible. 
We remark that it is much less natural to use $\widetilde{S}_{n}$ to construct various $K$ theory operations on $K(X)$ as done in Sect. 4 using a double cover $\widetilde{H}_{n}$ of the hyperoctahedral group.

The connection between $\mathcal{F}_{\Gamma}^{-}(X)$ and the $Q-\lambda$ ring in Sect. 5.4 does carry over to our new setup. Keeping Remark 5.3 in mind and knowing that $\widetilde{S}_{n}$ also has a so-called basic spin supermodule (cf. e.g. [Jo, FJW2]), we can use it to define the analog of (17). Indeed this also defines an analog of the map $\otimes^{n} \circ \aleph$ (cf. the diagram (14)), and thus an analog of $\varphi^{n} \circ \aleph$. Therefore, we have an analog of Proposition 5.2 in our new setup which generalizes Proposition 6.2 in [FJW2]. However there is an unpleasant square root of 2 in the formula which originates in the spin representation theory of $\widetilde{S}_{n}$ and $\widetilde{\Gamma}_{n}$. This is another reason why we have preferred the formulation in the main body of the paper using $\widetilde{H}_{n}$ and $\widetilde{H} \Gamma_{n}$.

One may wonder that why $K_{\widetilde{H} \Gamma_{n}}^{-}\left(X^{n}\right)$ and $K_{\widetilde{\Gamma}_{n}}^{-}\left(X^{n}\right)$ are so similar to each other and there are almost parallel constructions on $\mathcal{F}_{\Gamma}^{-}(X)$ and $\mathfrak{F}_{\Gamma}^{-}(X)$. When $X$ is a point and thus the $K$-groups reduces to the corresponding Grothendieck groups of spin supermodules, this has been noticed by various different authors (cf. e.g. [Ser, Jo2, Naz, Y, FJW2] and the references therein). Yamaguchi [Y] explains clearly such a phenomenon by establishing an isomorphism between the group superalgebra $\mathbb{C}\left[\widetilde{H}_{n}\right] /\langle z=-1\rangle$ and the ( $\mathbb{Z}_{2}$-graded) tensor product of the group superalgebra $\mathbb{C}\left[\widetilde{S}_{n}\right] /\langle z=-1\rangle$ with the complex Clifford algebra $C_{n}$ of $n$ variables (this is not the same copy of $C_{n}$ associated to the subgroup $\Pi_{n}$ in $\widetilde{H}_{n}$ !). It follows that the group superalgebra $\mathbb{C}\left[\widetilde{H} \Gamma_{n}\right] /\langle z=-1\rangle$ is isomorphic to the tensor product of the group superalgebra $\mathbb{C}\left[\widetilde{\Gamma}_{n}\right] /\langle z=-1\rangle$ with $C_{n}$. Note that a Clifford algebra admits a unique irreducible supermodule. As this $C_{n}$ acts on an $\widetilde{H} \Gamma_{n}$-bundle over $X^{n}$ fiberwise, this isomorphism provides a direct isomorphism between $K_{\widetilde{H} \Gamma_{n}}^{-}\left(X^{n}\right)$ and $K_{\widetilde{\Gamma}_{n}}^{-}\left(X^{n}\right)$.

We can also forget about the $\mathbb{Z}_{2}$-gradings (i.e. the super structures) in the group $\widetilde{\Gamma}_{n}$, in the construction of the category $\mathcal{C}_{\widetilde{\Gamma}_{n}}^{-}\left(X^{n}\right)$ and its associated $K$-group $K_{\widetilde{\Gamma}_{n}}^{-}\left(X^{n}\right)$. Let us denote the resulting new $K$-group by $K_{\widetilde{\Gamma}_{n}}^{s}\left(X^{n}\right)$. In particular when $X$ is a point and $\Gamma$ is trivial, this reduces to the Grothendick group $R^{s}\left(\widetilde{\Gamma}_{n}\right)$ of spin (not super) modules of $\widetilde{S}_{n}$ where $z$ still acts as -1 . We can then apply the decomposition theorem of Adem-Ruan $[\mathrm{AR}]$ to calculate $K_{\widetilde{\Gamma}_{n}}^{s}\left(X^{n}\right) \otimes \mathbb{C}$ in terms of $K_{\Gamma}(X) \otimes \mathbb{C}$. The difference here from the calculations in Theorem 5.2 and Theorem 6.2 is that the odd split conjugacy classes of $\widetilde{\Gamma}_{n}$ will also make contributions. Recall that the orbifold Euler number $e(X, \Gamma)$ defined in [DHVW] is the same as the Euler number of the equivariant $K$-theory $K_{\Gamma}(X)$. Using the description of even and odd split conjugacy classes of $\widetilde{\Gamma}_{n}$ (cf. [FJW2], Theorem 2.5), we can obtain the Euler number of $K_{\widetilde{\Gamma}_{n}}^{s}\left(X^{n}\right)$, denoted by $e^{s}\left(X^{n}, \widetilde{\Gamma}_{n}\right)$, in terms of the following generating function (compare Corollary 5.1): 


$$
\begin{aligned}
\sum_{n=0}^{\infty} t^{n} e^{s}\left(X^{n}, \widetilde{\Gamma}_{n}\right)= & \prod_{r=1}^{\infty}\left(1-t^{2 r-1}\right)^{-e(X, \Gamma)} \\
& +\prod_{r=1}^{\infty}\left(1+t^{2 r-1}\right)^{e(X, \Gamma)} . \\
& \cdot \frac{1}{2}\left(\prod_{r=1}^{\infty}\left(1+t^{2 r}\right)^{e(X, \Gamma)}-\prod_{r=1}^{\infty}\left(1-t^{2 r}\right)^{e(X, \Gamma)}\right) .
\end{aligned}
$$

The second summand in the right-hand side of the above equation counts the contributions from odd split conjugacy classes. When we set $X$ to be a point and $\Gamma$ trivial (and thus $e(X, \Gamma)=1$ ), this formula reduces to the classical generating function for the spin Grothendick group $R^{s}\left(\widetilde{S}_{n}\right)$ (cf. Theorem 3.6, [Jo], pp. 213; Corollary 3.10, $[\mathrm{HH}]$, pp. 32). We remark that due to some inaccurate analysis

of split conjugacy classes of $\widetilde{S}_{n}$, the formula (6.10) given in [Di] (even in the case when $\Gamma$ is trivial and $X$ is a point) is incompatible with this classical statement.

\section{References}

[AR] A. Adem and Y. Ruan, Twisted orbifold K-theory, math.AT/0107168.

[At] M. Atiyah, Power operations in K-theory, Quart. J. Math. Oxford 17 (1966), 165-193.

[AS] M. Atiyah and G. Segal, On equivariant Euler characteristics, J. Geom. Phys. 6 (1989) 671-677.

[BC] P. Baum and A. Connes, Chern character for discrete groups, In: Y. Matsumoto et al (eds.), A Fete of Topology, Academic Press, 1988.

[Bo] R. Borcherds, Vertex algebras, Kac-Moody algebras, and the Monster, Proc. Natl. Acad. Sci. USA 83 (1986), 3068-3071.

[Di] R. Dijkgraaf, Discrete torsion and symmetric products, hep-th/9912101.

[DHVW] L. Dixon, J.A. Harvey, C. Vafa, and E. Witten, Strings on orbifolds, Nuclear Phys. B 261 (1985), 678-686.

[FJW1] I. Frenkel, N. Jing and W. Wang, Vertex representations via finite groups and the McKay correspondence, Internat. Math. Res. Notices 4 (2000), 195-222, math.QA/9907166.

[FJW2] I. Frenkel, N. Jing and W. Wang, Twisted vertex representations via spin groups and the McKay correspondence, Duke Math. J. 111 (2002), 51-96, math.QA/0007159. 
[FLM] I. Frenkel, J. Lepowsky and A. Meurman, Vertex operator algebras and the Monster, Academic Press, New York 1988.

[Gr] I. Grojnowski, Instantons and affine algebras I: the Hilbert scheme and vertex operators, Math. Res. Lett. 3 (1996), 275-291.

$[\mathrm{HH}] \quad$ P. N. Hoffman and J. F. Humphreys, Projective representations of the symmetric groups: Q-functions and shifted tableaux, Clarendon Press, Oxford, 1992.

[HKR] M. Hopkins, N. Kuhn, and D. Ravenel, Generalized group characters and complex oriented cohomology theories, J. Amer. Math. Soc. 13 (2000), $553-594$.

$[\mathrm{J}] \quad$ N. Jing, Vertex operators, symmetric functions and the spin group $\Gamma_{n}$, J. Alg. 138 (1991), 340-398.

[JW] N. Jing and W. Wang, Twisted vertex representations and spin characters, Math. Z. 239 (2002), 715-746, math.QA/0104127.

[Jo] T. Józefiak, Characters of projective representations of symmetric groups, Expositiones Math. 7 (1989), 193-247.

[Jo1] T. Józefiak, Semisimple superalgebras, in: Some Current Trends in Algebra, Proc. of the Varna Conf. 1986, Lect. Notes in Math. 1352 (1988), 96-113.

[Jo2] T. Józefiak, A class of projective representations of hyperoctahedral groups and Schur Q-functions, in: Topics in Algebra, Banach Center Publications 26, Part 2, 317-326, PWN-Polish Scientific Publishers, Waszawa, 1990.

[Ka] M. Karoubi, Algèbres de Clifford et K-théorie, Ann. Scient. Éc. Norm. Sup., $4^{e}$ Série, t. 1 (1968), 161-270.

$[\mathrm{Ku}] \quad$ N. Kuhn, Character rings in algebraic topology, In: Advances in Homotopy, London Math. Soc. Lect. Notes Series 139 (1989), 111-126.

[Kn] D. Knutson, $\lambda$-rings and the representation of the symmetric group, Lect. Notes in Math. 308, Springer-Verlag, 1973.

[M] I. Macdonald, Symmetric functions and Hall polynomials, Second Edition, Oxford, Clarendon Press, 1995.

[Na] H. Nakajima, Lectures on Hilbert schemes of points on surfaces, Univ. Lect. Ser. 18, Amer. Math. Soc., 1999.

[Naz] M. Nazarov, Young's symmetrizers for projective representations of the symmetric group, Adv. in Math. 127 (1997), 190-257. 
[Ru] Y. Ruan, Stringy geometry and topology of orbifolds, math.AG/0011149.

[Sc] I. Schur, Über die Darstellung der symmetrischen und der alternierenden Gruppe durch gebrochene lineare Substitutionen, J. Reine Angew. Math. 139 (1911), 155-250.

[Seg1] G. Segal, Equivariant K-theory, Publ. Math. IHES, 34 (1968), 129-151.

[Seg2] G. Segal, Equivariant K-theory and symmetric products, 1996 Preprint.

[Ser] A. Sergeev, The tensor algebra of the identity representation as a module over the Lie superalgebras $g l(n, m)$ and $Q(n)$, Math. USSR Sbornik 51 (1985), 419-427.

[Sh] E. Sharpe, Recent developments in discrete torsion, Phys. Lett. B 498 (2001) 104-110, hep-th/0008191.

[Va] C. Vafa, Modular invariance and discrete torsion on orbifolds, Nucl. Phys. B273 (1986), 592-606.

[VW1] C. Vafa and E. Witten, A strong coupling test of S-duality, Nucl. Phys. B 431 (1994), 3-77.

[VW2] C. Vafa and E. Witten, On orbifolds with discrete torsion, J. Geom. Phys. 15 (1995), 189-214.

[W1] W. Wang, Equivariant K-theory, wreath products and Heisenberg algebra, Duke Math. J. 103 (2000), 1-23, math.QA/9907151.

[W2] W. Wang, Hilbert schemes, wreath products, and the McKay correspondence, Preprint, math.AG/9912104.

[W3] W. Wang, Algebraic structures behind Hilbert schemes and wreath products, Contemp. Math. (to appear), math.QA/0011103.

[Y] M. Yamaguchi, A duality of the twisted group algebra of the symmetric group and a Lie superalgebra, J. Alg. 222 (1999), 301-327.

[Z] A. Zelevinsky, Representations of finite classical groups. A Hopf algebra approach, Lect. Notes in Math. 869, Springer-Verlag, Berlin-New York, 1981.

Department of Math., North Carolina State University, Raleigh, NC 27695.

Current address: Department of Mathematics, University of Virginia, Charlottesville, VA 22904, U.S.A. Email: ww9c@virginia.edu 